\title{
A Solid-State NMR Study of the Antibacterial Drug Cefpodoxime Proxetil
}

\section{Krishna Dey}

Dr. Hari Singh Gour University

Manasi Ghosh ( $\nabla$ manasi.ghosh@bhu.ac.in )

Banaras Hindu University

\section{Research Article}

Keywords: Solid-State, Antibacterial Drug, Cefpodoxime Proxetil

Posted Date: January 13th, 2021

DOI: https://doi.org/10.21203/rs.3.rs-140419/v1

License: (c) (1) This work is licensed under a Creative Commons Attribution 4.0 International License. Read Full License 


\title{
A solid-state NMR study of the Antibacterial Drug Cefpodoxime proxetil
}

\author{
Krishna Kishor Dey ${ }^{1}$, Manasi Ghosh ${ }^{2 *}$ \\ 1. Department of Physics, Dr. Harisingh Gour Central University, Sagar-470003, Madhya- \\ Pradesh, India \\ 2. Physics Section, MMV, Banaras Hindu University, Varanasi-221005, Uttar-Pradesh, India \\ *Corresponding author: manasi.ghosh@bhu.ac.in
}

\begin{abstract}
The structure-activity relationship of various molecular moieties of cefpodoxime proxetil is described by measuring Chemical shift anisotropy (CSA) tensor, spin-lattice relaxation time and molecular correlation time at twenty one crystallographically different carbon nuclei sites. Cefpodoxime proxetil molecule is associated with three rings, the aminothiazole ring, $\beta$-lactam ring, and the dihydrothiazine ring, which provide stability to the drug molecule towards $\beta$-lactamases and increase the affinity of the drug to PBPs receptors. A large variation of CSA parameters and motional degrees of freedom are observed among carbon nuclei reside on these three rings, which implies that the electronic environment, molecular conformation, and molecular dynamics are altered substantially within the ring. The substitution at the $\mathrm{C} 7$ position of the $\beta$-lactam ring (like acyl side chain, oxime group, and aminothiazole ring) is responsible for semi-synthetic incorporation. It also influences the antibacterial activity and the binding affinity of the drug with $\beta$-lactamase. A huge variation of the spin-lattice relaxation time and molecular correlation time is observed in this region. These types of description of the structure-activity relationship of the antibacterial drug cefpodoxime proxetil will be beneficial for developing the advanced antibacterial drugs, and it will also be useful in NMR crystallography.
\end{abstract}




\section{Introduction:}

The $\beta$-lactam antibiotics exhibit antibacterial activity by inhibiting the cell membrane synthesis in microorganisms. The basic action method of the $\beta$-lactam antibiotics is to interrupt the transpeptidation process that combines the individual peptidoglycan components of the bacterial cell wall. ${ }^{1}$ These types of antibiotics are bind with the inner surface of the bacterial cell membrane, the penicillin-binding proteins (PBPs). The PBPs enzymes are necessary to assemble the bacterial cell wall and to reshape the cell wall during the growth and the division of the bacterial cell. The biochemical modification of the basic structure of the $\beta$-lactam (a four-membered $\beta$-lactam ring fused to a second ring) is incorporated to increase bacterial cell wall penetration and binding to PBPs. In penicillins (the $\beta$-lactam antibiotics), the $\beta$-lactam ring is fused to a five-membered thiazolidine ring, whereas in cephalosporins, the $\beta$-lactam ring is attached to a six-membered dihydrothiazine ring. The cephalosporin is more active against $\beta$-lactamase-producing bacteria compared to penicillin. ${ }^{1}$ It also offer more sites for biochemical modifications. Therapeutically important modifications have been made at the acyl group attached to the $\beta$-lactam ring and the moiety attached with the dihydrothiazine ring. Modifications in the acyl side chain change the antibacterial activity, while modifications of the dihydrothiazine ring side chain alter the pharmacokinetics and metabolic parameters of the antibiotics. Cefpodoxime proxetil is a third-generation cephalosporin antibacterial drug. It is used for the treatment of infections due to Gram-positive and Gram-negative bacteria. It showed great efficacy to treat skin and soft tissue infections, acute otitis media, pharyngitis, bronchitis, pneumonia, respiratory and urinary tracts infection, and primarily gastrointestinal tract disturbances of pediatric patients. ${ }^{2}$ The current work is targeted to determine the structure-activity relationship of cefpodoxime proxetil by solid sate NMR spectroscopic measurements. High-resolution solid state NMR spectroscopy is an indispensable tool to determine the information about local electronic 
structure and symmetries encoded in chemical shift anisotropy (CSA) interactions. The detailed structure and molecular dynamics at various molecular moieties of cefpodoxime proxetil is portrayed by measuring principal components of CSA tensor at carystallographically different carbon nuclei sites by two-dimensional phase adjusted spinning sideband (2DPASS) cross-polarization (CP) magic angle spinning (MAS) solid-state NMR experiment. ${ }^{32,33}$ The dynamics at crystallographically different carbon nuclei sites are determined by site-specific spin-lattice relaxation time measurements by the Torchia CP method. ${ }^{47}$ The molecular correlation time at various carbon nuclei sites is also calculated.

3-18 It has none components. It can be diagonalized in the principal axis system (PAS) and the diagonal components are called principal components of chemical shift anisotropy tensor. Each element of the CSA tensor is composed of two components $\delta=$ $\delta^{d}+\delta^{p}$. The first component is the diamagnetic contribution arises when the electrons are in the ground state and the circulating current generates an induced magnetic field opposite to the direction of the external magnetic field. These components can be expressed as ${ }^{19}$

$$
\delta_{\gamma \gamma}^{d}=\frac{\mu_{0}}{4 \pi} \frac{e^{2}}{2 m_{e}}\left|\psi_{0}\right| \sum_{i} \frac{\alpha_{i}^{2}+\beta_{i}^{2}}{r_{i}^{3}}\left|\psi_{0}\right\rangle
$$

The second component is the paramagnetic contribution arises due the presence of the electrons in the excited state ${ }^{19}$

$$
\delta_{\alpha \beta}^{p}=-\frac{\mu_{0}}{4 \pi} \frac{e^{2}}{2 m_{e}} \sum_{k \neq 0}\left[\frac{\left.\left\langle\psi_{0}\left|\sum_{i} \widehat{l_{l \alpha}}\right| \Psi_{\mathrm{k}}\right\rangle\left|\psi_{\mathrm{k}}\right| \sum_{i} \frac{\widehat{l_{l \beta}}}{r_{i}^{3}}\left|\Psi_{0}\right\rangle+C . C\right]}{E_{k}-E_{0}}\right]
$$

Where $\alpha, \beta, \gamma$ denote the Cartesian coordinates. The $\psi$ are the electronic wavefunctions; $\psi_{0}$ and $\psi_{\mathrm{k}}$ are the ground state and the excited state electronic wavefunction respectively. $\alpha_{i}, \beta_{i}$ are the coordinates of the $i$ th electron along the $\alpha$ and $\beta$ directions respectively. $\widehat{l_{l \alpha}}, \widehat{l_{l \beta}}$ are 
the components of the electronic orbital angular momentum operator along the $\alpha$ and $\beta$ directions respectively. $E_{k}, E_{0}$ are the energy of the electron in the excited state and the ground state respectively. ${ }^{19}$

The detailed picture of the correlation between the structure and dynamics of charge transferred cocrystal, biopolymer, drug molecules, and glass, were given by 2DPASS CPMAS SSNMR experiment and Torchia CP experiment. ${ }^{34-46,57,58}$ The structure-activity relationship of cefpodoxime proxetil are investigated by 2DPASS CP-MAS SSNMR experiment $^{32,33}$ and Torchia-CP experiment, ${ }^{47}$ which will enlighten the path of developing the advanced antibacterial drug, and will be useful in NMR crystallography.

\section{Experimental:}

\subsection{NMR Measurements}

Active pharmaceutical ingredient of cefpodoxime proxetil was purchased from Sigma Aldrich. ${ }^{13} \mathrm{C}$ CP-MAS SSNMR, ${ }^{13} \mathrm{C}$ spin-lattice relaxation (by Torchia CP method ${ }^{47}$ ), and 2DPASS CP-MAS SSNMR experiments were performed on a JEOL ECX 500 NMR spectrometer, associated with $3.2 \mathrm{~mm}$ JEOL double-resonance MAS probe. ${ }^{13} \mathrm{C} C \mathrm{CP}-\mathrm{MAS}$ and Torchia CP experiments ${ }^{47}$ were performed at MAS speed $10 \mathrm{kHz}$ at room temperature. For the CP-MAS experiment, the contact time was $2 \mathrm{~ms}$ with SPINAL-64 ${ }^{1} \mathrm{H}$ decoupling. The

number of scans for ${ }^{13} \mathrm{C}$ CP MAS experiment scan was 32768 and for ${ }^{13} \mathrm{C}$ 2DPASS CP-MAS experiment number of scans was 4030 . For measuring ${ }^{13} \mathrm{C}$ relaxation by the Torchia $\mathrm{CP}$ method, the number of scans was 2048. All the experiments were performed at room temperature. The referencing for the ${ }^{13} \mathrm{C}$ spectrum is done by using tetramethylsilane.

\subsection{CSA Measurements}


The resonances lines in liquid state NMR spectra are very narrow because the anisotropic parts of the chemical shift, dipole-dipole, and the quadrupolar interactions are averaged out due to the rapid tumbling motions of the molecules. On the contrary, the resonance lines in the solid-state NMR spectra are considerably broadened due to the presence of all the anisotropic interactions. The broadening due to chemical shift anisotropy interaction can be removed when the magic angle spinning (MAS) frequency exceeds the span of the chemical shift anisotropy. But the information about the electronic distribution and molecular conformation is lost as those informations are encoded in CSA interaction. One option to retrieve the CSA information is to reduce the MAS frequency less than the span of the chemical shift anisotropy. In this condition, the solid-state NMR spectrum is flanked on both sides of the isotropic chemical shift by sidebands equally spaced at the MAS frequency. The intensities of these spinning sidebands are correlated with the CSA. Herzfeld and Berger had derived graphical and numerical methods for evaluating the principal components of the CSA tensor from the intensities of the sidebands. ${ }^{56}$

The 2DPASS CP-MAS SSNMR experiment is a useful technique to simplify the solidstate MAS NMR spectrum containing complicated spinning sideband manifolds. The pulse sequence consists of five $\pi$-pulses, separates spinning sidebands by their order. The duration of five $\pi$-pulses remains unchanged throughout the experiment. The time interval among five $\pi$-pulses is followed by PASS-equation and it was reported by Antzutkin et al. ${ }^{33}$ This experiment correlates the isotropic dimension with the anisotropic dimension. The 2D spectrum yields an infinite spinning speed spectrum with no sideband. The 2DPASS CPMAS SSNMR experiment works at lower MAS frequencies. For cefpodoxime proxetil, the 2DPASS CP-MAS SSNMR experiments were performed at MAS frequencies $600 \mathrm{~Hz}$ and $2 \mathrm{kHz} .90^{0}$ pulse length for the ${ }^{13} \mathrm{C}$ nucleus was 3.3 us. The Relaxation delay was $10 \mathrm{~s}$. The number of scans for the 2D PASS CP-MAS SSNMR experiments were 4030 (integral 
multiple of 13). The coherence transfer pathway for the 2D PASS NMR experiment was reported by Ghosh et al. ${ }^{34}$ Thirteen steps cogwheel phase cycling was used. Sixteen data points were acquired in the indirect dimension as the numbers of sidebands were less than sixteen. The anisotropic part of the chemical shift interaction in natural abundance ${ }^{13} \mathrm{C}$ spin$1 / 2$ nuclei (for those nuclei where the homonuclear dipole-dipole coupling is much less than the rotor frequency) evolves during the PASS sequence under the rotor pitch evolution in $\mathrm{t} 1$ dimension.

\section{Result and Discussion}

\subsection{Solid State NMR Spectral Analysis:}

Cefpodoxime proxetil is an ester modified prodrug of cefpodoxime. It is composed of aminothiazole ring, oxime group, acyl side chain, beta-lactam ring, dihydrothiazine ring, and ester group side chain. The beta-lactam ring is the core structure of antibiotics like cephalosporins, penicillins, carbapenems, and monobactams, which are also known as $\beta$ lactam antibiotics. The $\beta$-lactam antibiotics are worked by inhibiting the cross-linking of the peptidoglycan layer, which is an important component for preserving cell wall structural integrity of Gram-positive bacteria. The $\beta$-lactam ring is merged with the dihydrothiazine ring. ${ }^{19-21}$ Cephalosporins resemble penicillin as they both have a $\beta$-lactam ring, but the fivemember thiazolidine ring of penicillin is replaced by a six-member dihydrothiazine ring in cephalosporins. Cephalosporin is familiar as 7-aminocephalosporanic acid. The group substitution occurs at both sides of the 7-aminocephalosporanic acid.

Figure 1(a) shows the ${ }^{13} \mathrm{C}$ CP-MAS SSNMR spectrum of cefpodoxime proxetil. Isotropic chemical shift is large for those carbon atoms bonded with electronegative oxygen and nitrogen atoms. The electronegative atom attracts the electron cloud surrounding the nucleus. As a consequence, the effective magnetic field experienced by the nucleus is 
enhanced due to the lowering of the nuclear shielding effect, and the Larmor precession frequency of the corresponding nucleus is shifted towards the higher frequency region. The electronegative atoms give acid stability to the drug molecule. The polar group extends the spectrum of antibacterial activity. The isotropic, as well as the anisotropic chemical shift of the polar group carbon nuclei $\mathrm{C} 15$, is very high. 

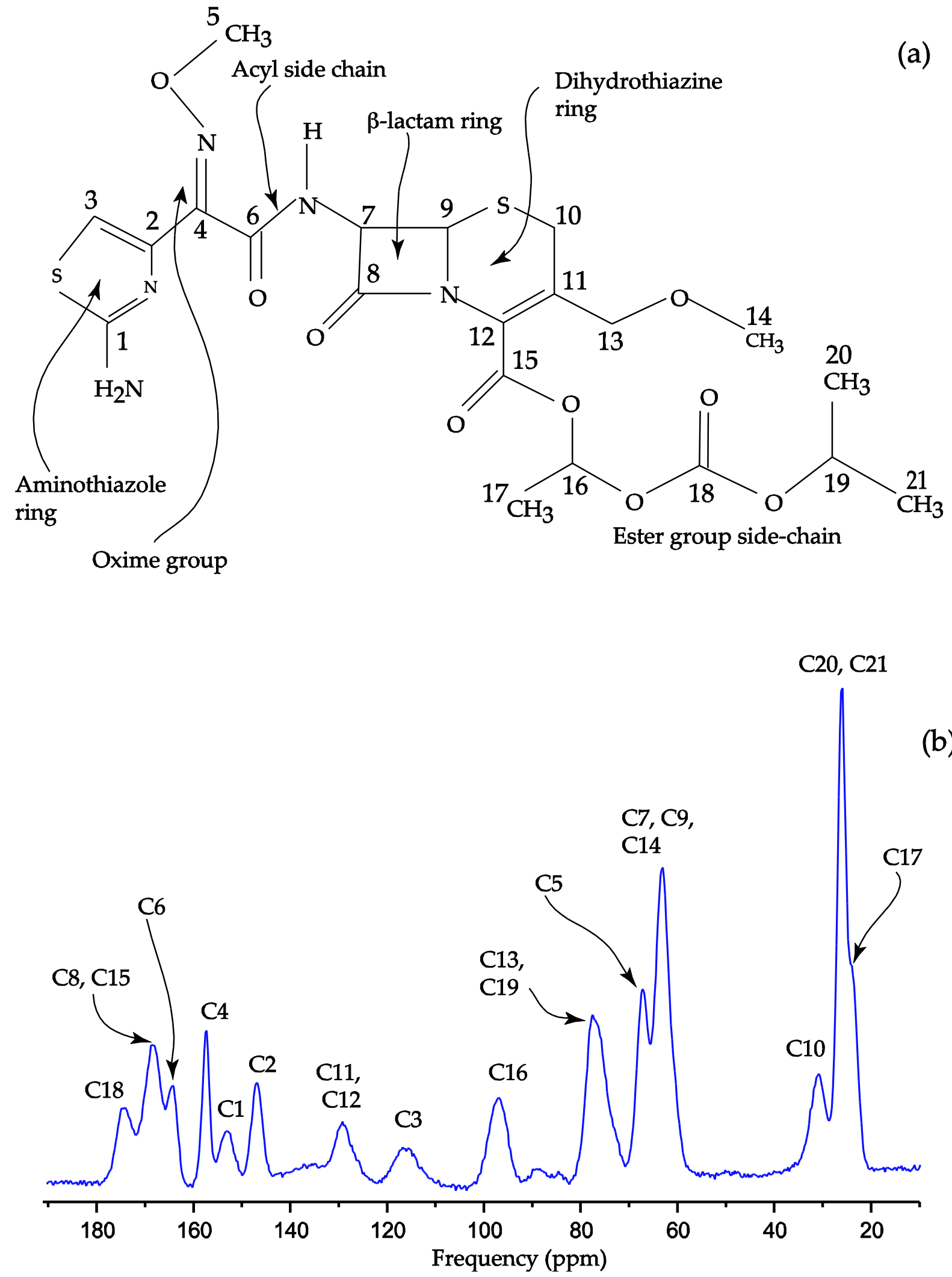

Figure 1: (a) The chemical structure of cefpodoxime proxetil, (b) ${ }^{13} \mathrm{C}$ CP-MAS SSNMR spectrum of cefpodoxime proxetil. 


\subsection{CSA Measurements:}

The values of the principal components of the chemical shift anisotropy tensor depend on the distribution of electron density around nuclei. The electron density distribution is correlated with the geometry of chemical bonds, i.e. with the structure of the molecular moiety. The chemical shift anisotropy tensor can be represented by an ellipsoid centred on the nucleus it applies to. The axes of the ellipsoid coincide with the axes of the PAS of the CSA tensor. The length of each principal axis of the ellipsoid is proportional to the principal components of the CSA tensor associated with that principal axis. Hence, the orientation of the CSA tensor changes with the orientation of the molecule. ${ }^{19}$ The CSA tensor at a nuclear site is axially symmetric in PAS if the principal axis coincides with the symmetry axis and the principal components of the CSA tensor is such that $\delta_{11}=\delta_{22}$. Table 1 shows that the spinning CSA sideband pattern of $\mathrm{C} 4, \mathrm{C} 5$, and C6 nuclei is axially symmetric.

Isotropic

chemical shift $\left(\delta_{\text {iso }}=\frac{\delta_{11}+\delta_{22}+\delta_{33}}{3}\right)$ is defined as the center of gravity of the spinning CSA sideband pattern. ${ }^{23}$ According to the Haeberlen convention anisotropy parameter is defined $\operatorname{as}\left(\Delta \delta=\delta_{33}-\frac{\left(\delta_{11}+\delta_{22}\right)}{2}\right)$, and the asymmetry parameter is defined as $\left(\eta=\frac{\delta_{22}-\delta_{11}}{\delta_{33}-\delta_{i s o}}\right)$. The chemical shift frequency can be expressed as

$$
\omega(\theta, \Phi)=-\omega_{0}\left(\delta_{11} \sin ^{2} \theta \cos ^{2} \Phi+\delta_{22} \sin ^{2} \theta \sin ^{2} \Phi+\delta_{33} \cos ^{2} \theta\right)
$$

Where $\theta$, and $\Phi$ are the polar and azimuthal angles with respect to the direction of the applied magnetic field $\left(\mathbf{B}_{\mathbf{0}}\right)$ in the principal axis system (PAS). All values of the angle $\theta$, and $\Phi$ are possible in a powder sample. Each different molecular orientation implies a different orientation of PAS with respect to the external magnetic field as the PAS is fixed in the molecule. A different chemical shift is associated with each orientation of the molecule. Therefore, the spectrum takes the shape of a powder pattern with lines from the different 
molecular orientations. The intensity at a particular frequency is proportional to the number of molecular orientations with a particular chemical shift. The shape of the powder pattern depends on the symmetry of the CSA tensor i.e. on the symmetry surrounding the nucleus.

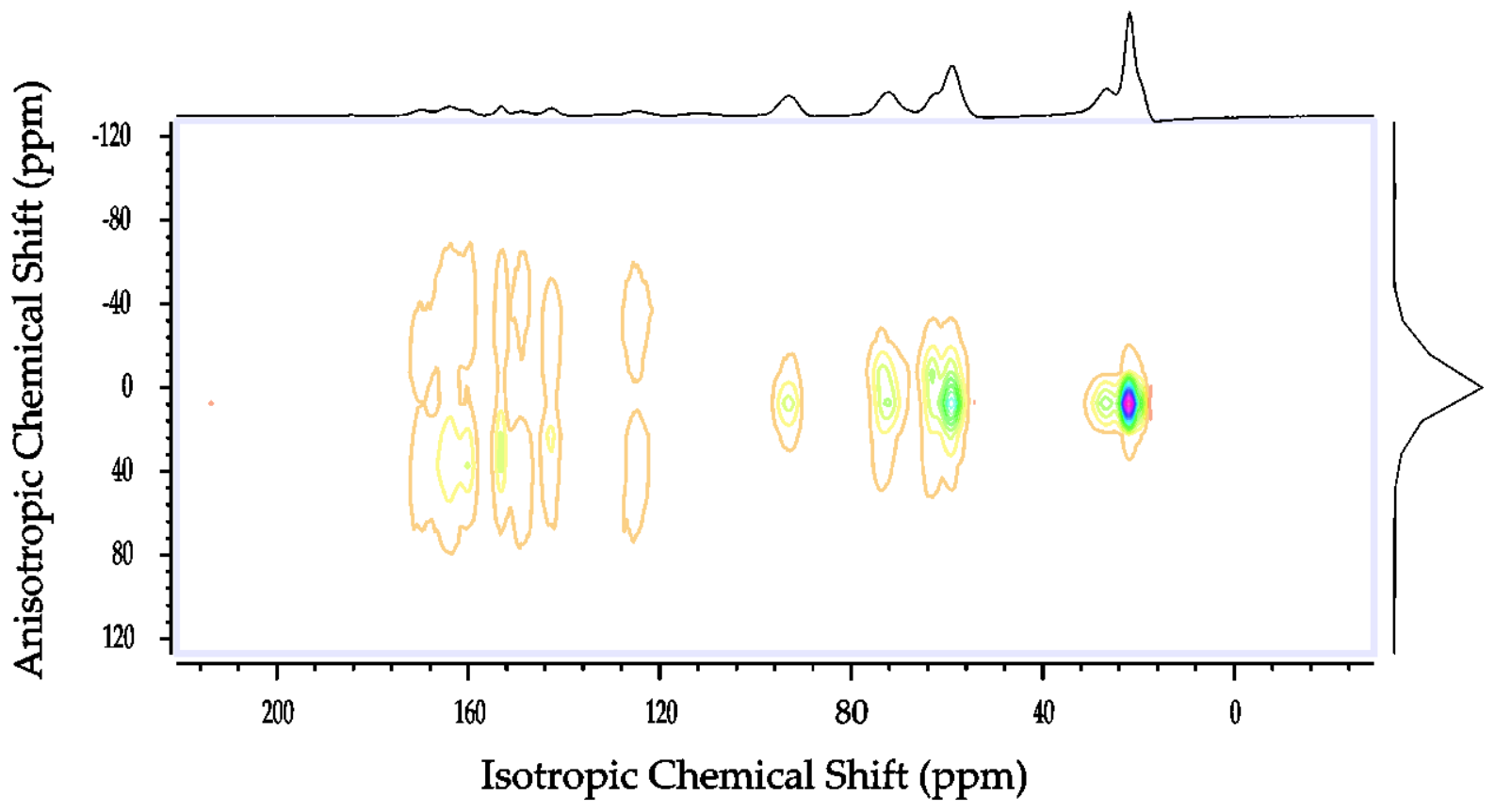


Figure 2: 2DPASS CP-MAS NMR spectrum of cefpodoxime proxetil. The direction dimension represents the pure isotropic spectrum of cefpodoxime proxetil with zero sideband.
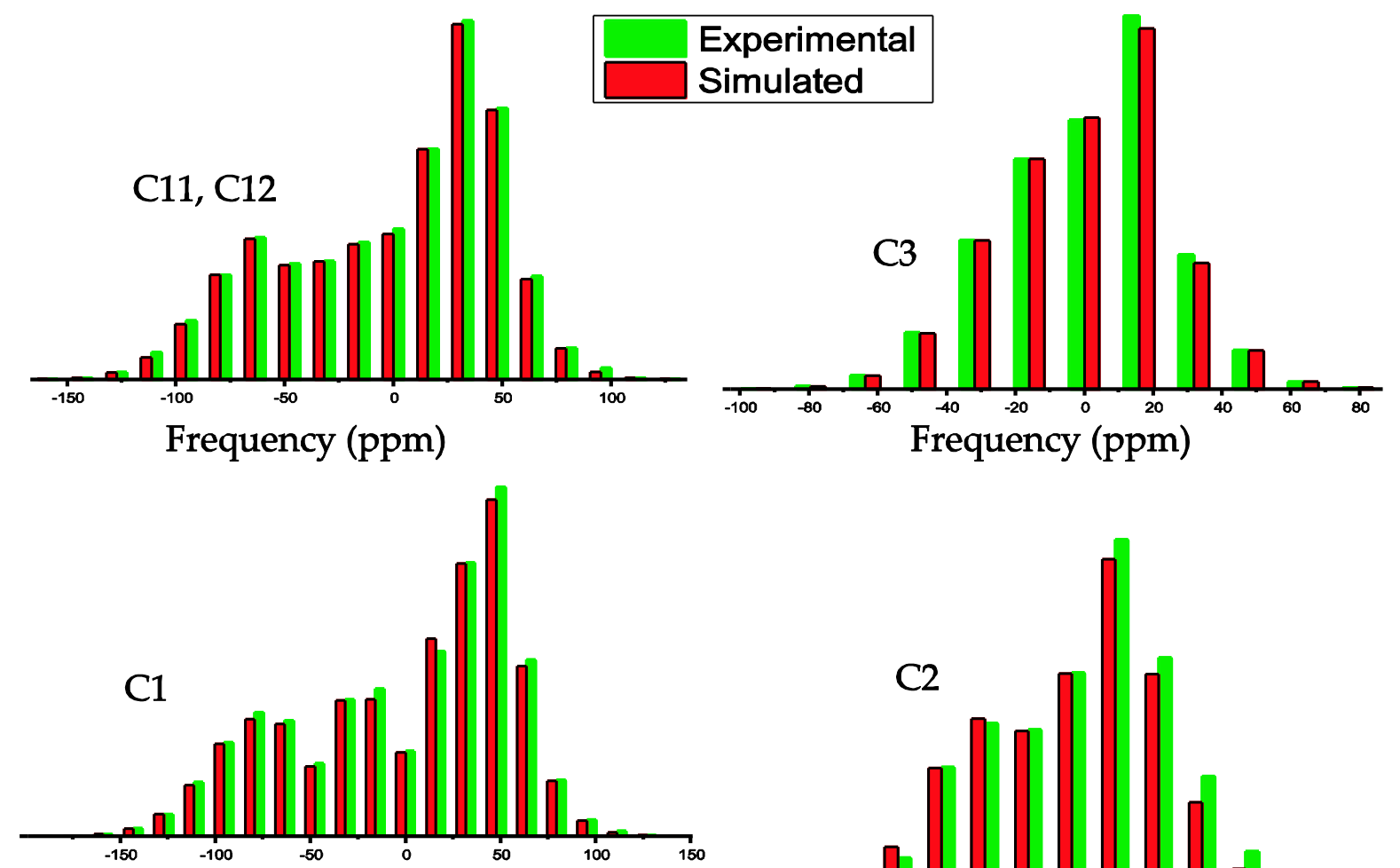

Frequency (ppm)

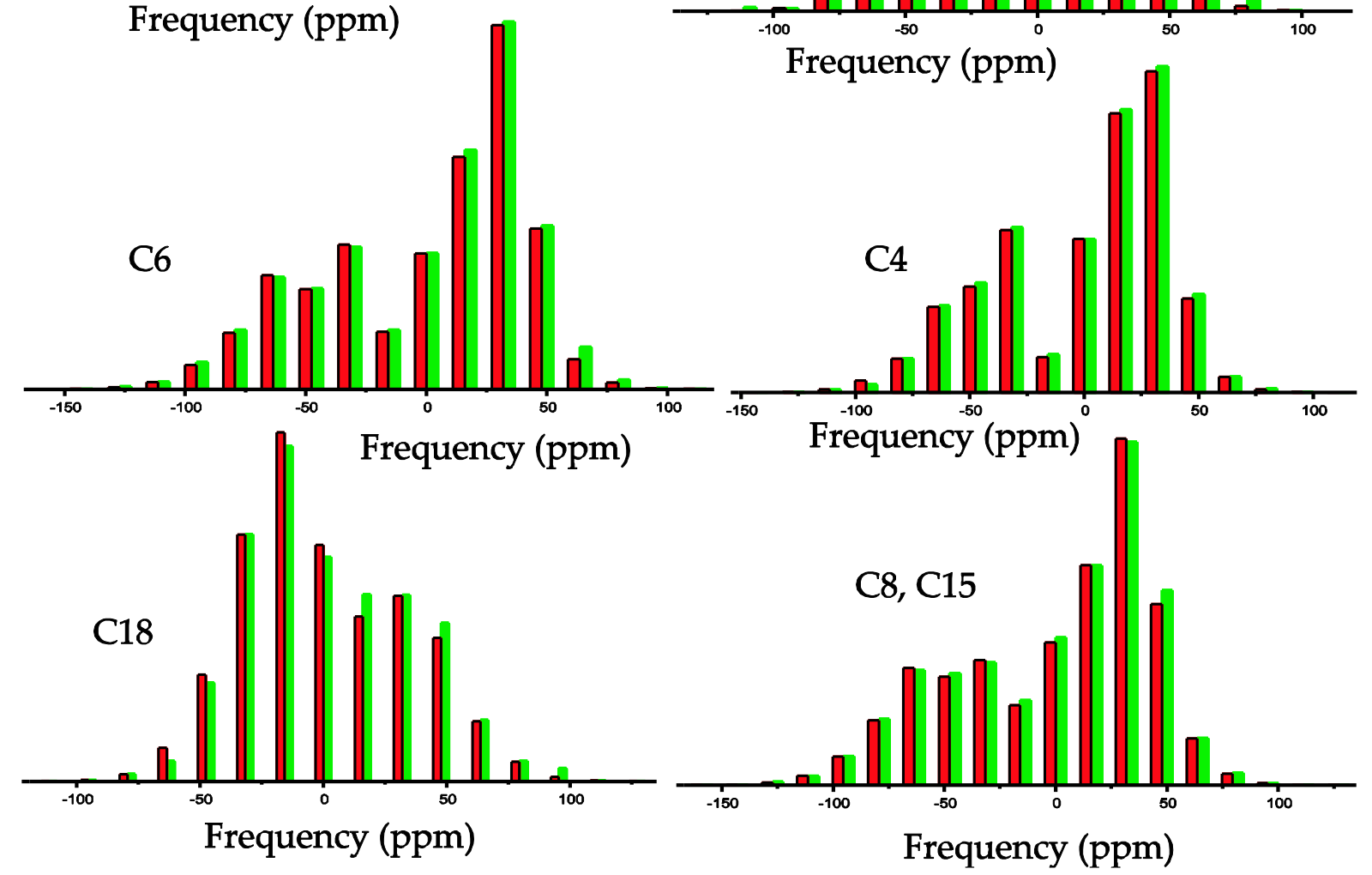

Figure 3: Spinning CSA sideband pattern of cefpodoxime proxetil. 

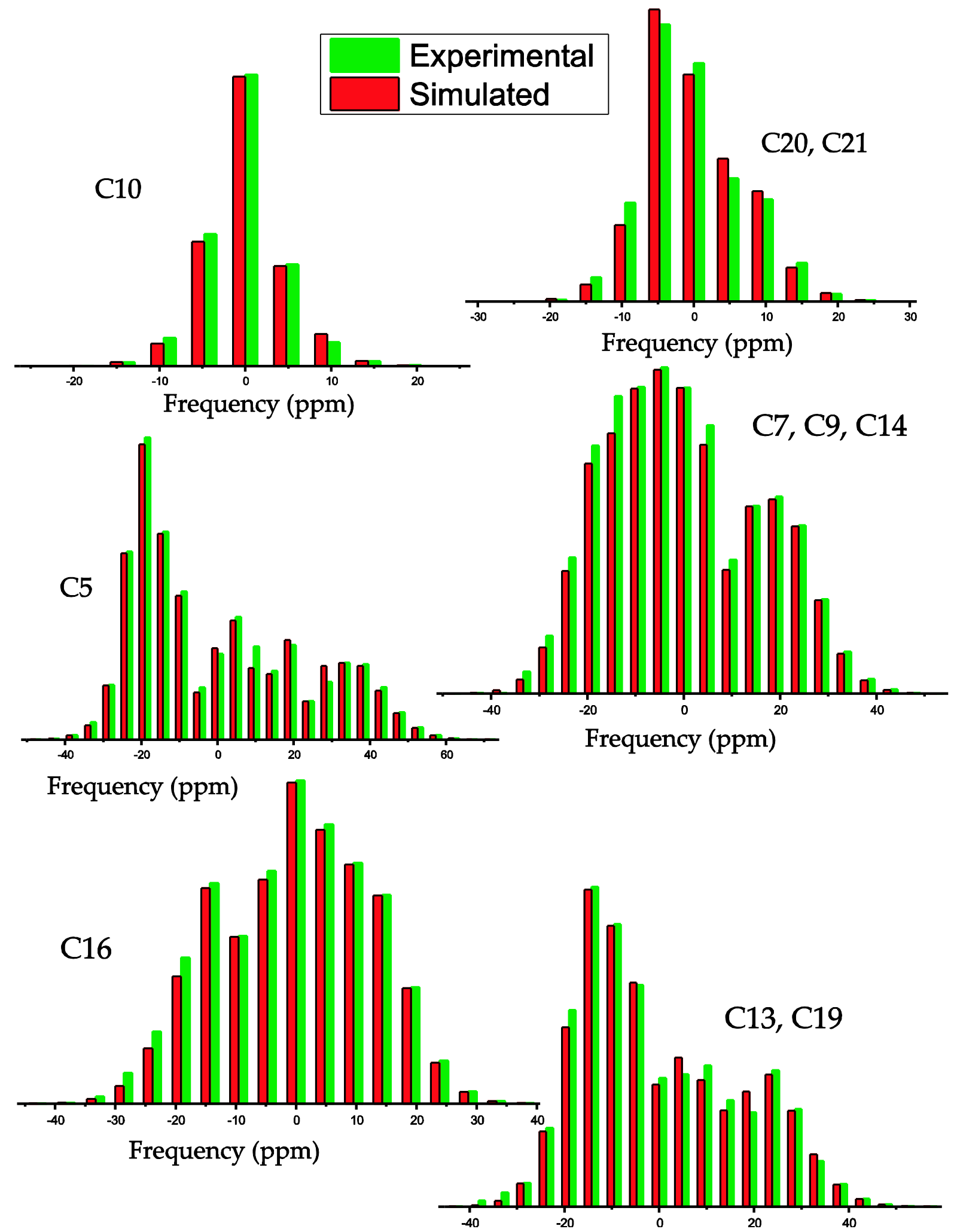

Figure 4: Spinning CSA sideband pattern of cefpodoxime proxetil. 
Table 1: The chemical shift anisotropy parameters of cefpodoxime proxetil at crystallographically different sites.

\begin{tabular}{|c|c|c|c|c|c|c|}
\hline \multicolumn{7}{|c|}{ CSA parameters of Cefpodoxim proxetil } \\
\hline $\begin{array}{l}\text { Carbon } \\
\text { from } \\
\text { differe } \\
\text { nt } \\
\text { chemic } \\
\text { al } \\
\text { environ } \\
\text { ment }\end{array}$ & $\begin{aligned} \delta_{11} \\
(\mathrm{ppm})\end{aligned}$ & $\begin{array}{c}\delta_{22} \\
(\mathrm{ppm})\end{array}$ & $\begin{array}{c}\delta_{33} \\
(\mathrm{ppm})\end{array}$ & $\begin{array}{c}\delta_{\text {iso }} \\
(\mathrm{ppm})\end{array}$ & $\begin{array}{l}\text { Anisotropy } \\
(\mathrm{ppm}) \\
\Delta \delta \\
=\delta_{33} \\
-\frac{\left(\delta_{11}+\delta_{22}\right)}{2}\end{array}$ & $\begin{array}{l}\text { Asymmetry } \\
\eta=\frac{\delta_{22}-\delta_{11}}{\delta_{33}-\delta_{i s o}}\end{array}$ \\
\hline C18 & $240.9 \pm 4$ & $157.4 \pm 2.5$ & $112.7 \pm 3.2$ & 170.3 & $105.8 \pm 6.1$ & $0.6 \pm 0.07$ \\
\hline $\begin{array}{l}\mathrm{C} 8 \\
\mathrm{C} 15\end{array}$ & $224 \pm 4.1$ & $201.5 \pm 2.7$ & $67.8 \pm 3.1$ & 164.4 & $-144.9 \pm 4.7$ & $0.2 \pm 0.05$ \\
\hline C6 & $207.7 \pm 3.1$ & $207.7 \pm 2$ & $66.9 \pm 2.3$ & 160.8 & $-140.9 \pm 3.5$ & 0 \\
\hline $\mathrm{C} 4$ & $195.6 \pm 1.8$ & $195.6 \pm 1.2$ & $69.9 \pm 1.4$ & 153.7 & $-125.7 \pm 2.1$ & 0 \\
\hline $\mathrm{C} 1$ & $226.9 \pm 6.9$ & $195.9 \pm 4.7$ & $25.1 \pm 5.1$ & 149.3 & $-186.3 \pm 7.7$ & $0.2 \pm 0.07$ \\
\hline $\mathrm{C} 2$ & $199.9 \pm 2.9$ & $155.4 \pm 1.8$ & $75 \pm 2.3$ & 143.4 & $-102.7 \pm 3.5$ & $0.6 \pm 0.06$ \\
\hline $\begin{array}{l}\text { C11,C1 } \\
2\end{array}$ & $195.6 \pm 7.2$ & $161.6 \pm 4.8$ & $18.9 \pm 5.4$ & 125.4 & $-159.7 \pm 8.1$ & $0.3 \pm 0.08$ \\
\hline $\mathrm{C} 3$ & $155.8 \pm 3.6$ & $119.8 \pm 2.1$ & $61.6 \pm 2.9$ & 112.4 & $-76.1 \pm 4.4$ & $0.7 \pm 0.09$ \\
\hline C16 & $115.5 \pm 0.7$ & $94.2 \pm 0.4$ & $67.4 \pm 0.5$ & 92.4 & $-37.4 \pm 0.8$ & $0.8 \pm 0.04$ \\
\hline $\begin{array}{l}\text { C13, } \\
\text { C19 }\end{array}$ & $109.6 \pm 0.7$ & $61.1 \pm 0.4$ & $47.8 \pm 0.5$ & 72.8 & $55.2 \pm 1$ & $0.4 \pm 0.02$ \\
\hline $\mathrm{C} 5$ & $113.3 \pm 1.4$ & $39.1 \pm 0.9$ & $37.4 \pm 1$ & 63.3 & $75 \pm 2.1$ & 0.03 \\
\hline $\begin{array}{l}\text { C7, C9, } \\
\text { C14 }\end{array}$ & $91.9 \pm 1.5$ & $53.7 \pm 1$ & $31.2 \pm 0.1$ & 58.9 & $49.4 \pm 2.2$ & $0.7 \pm 0.05$ \\
\hline $\mathrm{C} 10$ & $35.7 \pm 0.1$ & $25 \pm 0.06$ & $17.9 \pm 0.08$ & 26.2 & $14.2 \pm 0.1$ & 0.7 \\
\hline $\begin{array}{l}\mathrm{C} 20, \\
\mathrm{C} 21\end{array}$ & $36.5 \pm 0.2$ & $18.2 \pm 0.1$ & $10.7 \pm 0.2$ & 21.8 & $22 \pm 0.3$ & $0.5 \pm 0.01$ \\
\hline
\end{tabular}

Huge variations of the CSA parameters are observed for the carbon nuclei reside on $\beta$-lactam ring. The CSA parameter of $\mathrm{C} 8$ nuclei is much higher than $\mathrm{C} 7$ and $\mathrm{C} 9$ nuclei. From Table 2, it is clear that the molecular correlation time of C8 nuclei is $1.6 \times 10^{-4} \mathrm{~s}$, where it is $1.0 \times$ $10^{-5}$ s for $\mathrm{C} 7$ and $\mathrm{C} 9$. Hence, the motional degrees of freedom are also varied within the fourmember $\beta$-lactam ring. 

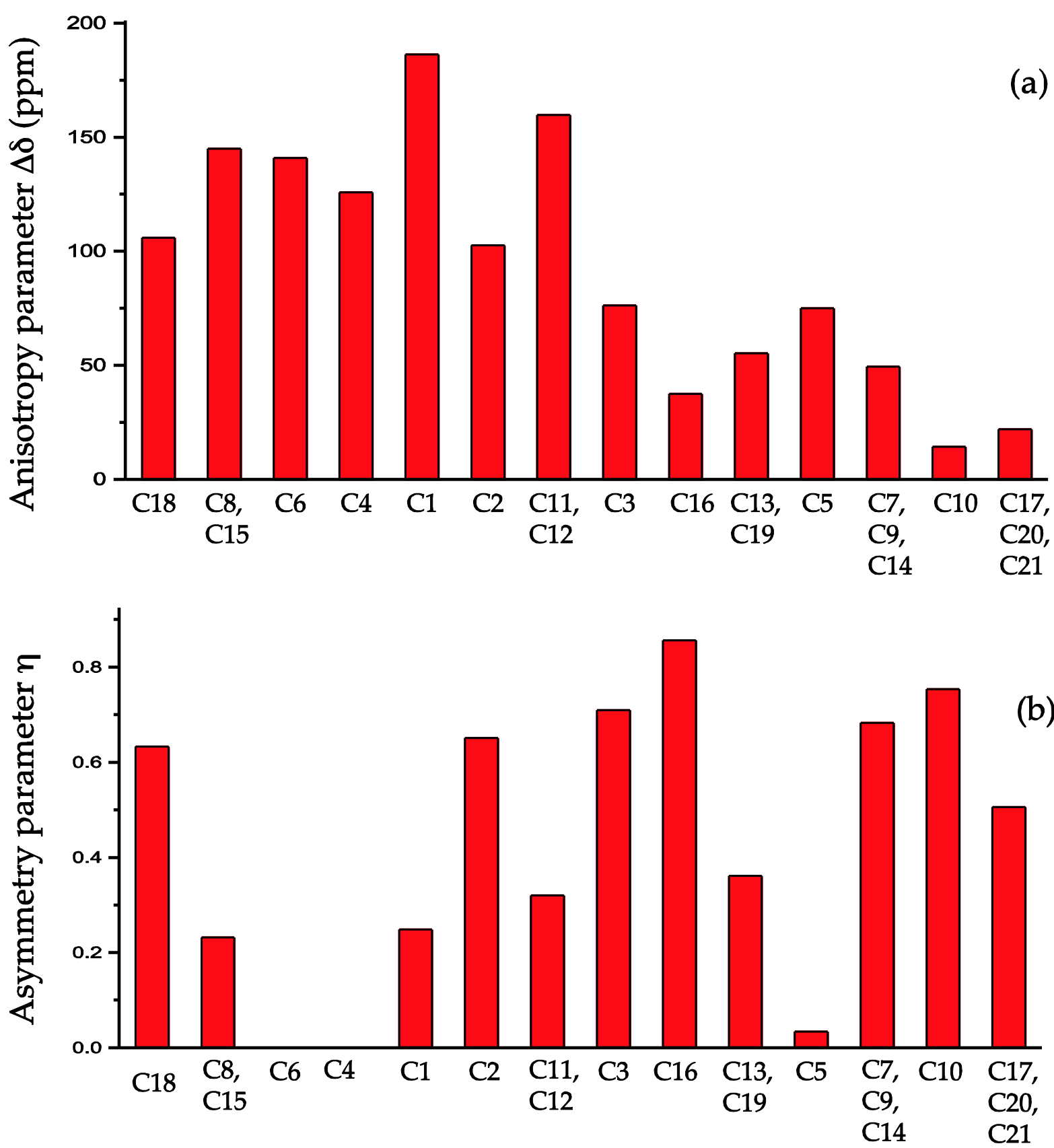

Figure 5: The bar-diagram of (a) anisotropy parameter and (b) asymmetry parameter of cefpodoxime proxetil at crystallographically different carbon sites.

According to the Haeberlen convention, anisotropy parameter and asymmetry $(\eta)$ parameter are defined as $\Delta \delta=\delta_{33}-\frac{\left(\delta_{11}+\delta_{22}\right)}{2}$ and $\eta=\frac{\delta_{22}-\delta_{11}}{\delta_{33}-\delta_{i s o}}$. The value of the anisotropy parameter defines the largest separation of the spinning CSA sideband pattern from the center of gravity, and the sign of the anisotropy parameter says on which direction of the center of 
gravity one can find the largest separation. Asymmetry parameter measures how much the spinning CSA sideband pattern deviates from its axially symmetric shape. Figure 5 shows the bar-diagram of (a) anisotropy parameter and (b) asymmetry parameter at crystallographically different sites of cefpodoxime proxetil. If $\delta_{11}=\delta_{22}$ or $\delta_{11}=\delta_{33}$, then the value of the asymmetry parameter is zero, and the shape of the spinning CSA sideband pattern is axially symmetric. Figure 5(b), Figure 3, Figure 4, and Table 1 show that the CSA pattern of C4, C5, and C6 is axially symmetric. On the contrary, the CSA pattern is highly asymmetric for C10, C16, C2, C3, C18, C7, C9, C14, C17, C20, and C21. The spinning CSA sideband pattern for $\mathrm{C} 1, \mathrm{C} 8$, and $\mathrm{C} 15$ is nearly axially symmetric.

Table 1 shows the CSA parameters are very large for $\mathrm{C} 18, \mathrm{C} 8, \mathrm{C} 15, \mathrm{C} 6$, and C4 bonded with electronegative oxygen and nitrogen atoms. The presence of an electronegative atom surrounding the nucleus decrease the shielding effect by attracting the electron cloud surrounding the nucleus. Consequently, the effective magnetic field experienced by the nucleus is increased and Larmor precession frequency shifted towards the higher frequency side. The magnetic anisotropy is another reason for the large values of CSA parameters for the carbonyl group carbons $\mathrm{C} 18, \mathrm{C} 8, \mathrm{C} 15$, and C6. There is no centre of symmetry for carbonyl group carbon. Hence, there appear three different magnetic susceptibilities $\left(x_{x}, x_{y}, x_{z}\right)$ along three mutually perpendicular directions in the principal axes system (PAS). As a consequence, there appear two anisotropic susceptibilities - one parallel to the magnetic field $\left(\Delta x_{\|}=x_{z}-x_{x}\right)$ and another perpendicular to the magnetic field $\left(\Delta x_{\perp}=\right.$ $\left.x_{y}-x_{x}\right)$. The McConnell equation ${ }^{53}$ of magnetic anisotropy for non-symmetric carbonyl group is

$$
\delta_{\text {anis }}=\left\{\Delta X_{\|}\left(3 \cos ^{2} \theta_{1}-1\right)+\Delta X_{\perp}\left(3 \cos ^{2} \theta_{2}-1\right)\right\} / 3 R^{3}
$$


Where $\theta_{1}$ is the angle between the radius vector and $x$-axis and $\theta_{2}$ is the angle between radius vector and $z$-axis. ${ }^{54}$ The anisotropic magnetic susceptibility is the source of the directional dependent magnetic field, which manifests as large values of chemical shift anisotropy at carbonyl site. The carbonyl group contains a polar bond. Hence, there arises a polarization on the electron cloud surrounding the carbon nucleus due to the electrostatic interaction of the central molecule with other molecules. The strength of the induced magnetic field is different at different directions due to this polarization, which is also a reason behind the large CSA parameters of carbonyl group carbon. ${ }^{54}$

The aminothiazole ring, $\beta$-lactam ring, dihydrothiazine ring brings stability towards the most commonly found plasmid-mediated $\beta$-lactamases and increase the affinity of the drug to the penicillin binding proteins (PBPs) receptors. ${ }^{2}$ The anisotropy parameter $(\Delta \delta=$ $\left.\delta_{33}-\frac{\left(\delta_{11}+\delta_{22}\right)}{2}\right)$ of $\mathrm{C} 1$ carbon nuclei reside on aminothiazole ring is much higher than $\mathrm{C} 2$ and $\mathrm{C} 3$ nuclei reside on the same ring. All three carbon atoms $\mathrm{C} 1, \mathrm{C} 2$ and $\mathrm{C} 3$ are in sp2 hybridized state. The spinning CSA sideband pattern of $\mathrm{C} 1$ is nearly axially symmetric, whereas the CSA pattern of $\mathrm{C} 2$ and $\mathrm{C} 3$ are highly asymmetric. The CSA parameters of C8 nuclei of $\beta$-lactam ring are much larger than $\mathrm{C} 7$ and $\mathrm{C} 9$ nuclei on the same ring. The spinning CSA sideband pattern of C8 is nearly axially symmetric, but the spinning CSA pattern of $\mathrm{C} 7$ and $\mathrm{C} 9$ are asymmetric. $\mathrm{C} 8$ are in sp2 hybridized state and $\mathrm{C} 7, \mathrm{C} 9$ are in sp3 hybridized state. The CSA parameters of C11, C12 nuclei of dihydrothiazine ring are much higher than $\mathrm{C} 10$ of the same ring. $\mathrm{C} 11, \mathrm{C} 12$ are in $\mathrm{sp} 2$ hybridized state, and $\mathrm{C} 10$ is in $\mathrm{sp} 3$ hybridized state. The spinning CSA sideband pattern of C11 and C12 are nearly axially symmetric and $\mathrm{C} 10$ is highly asymmetric. In these three rings, large variation of CSA parameters of carbon atoms is observed, which implies that the electronic environment and molecular conformation are hugely varied within the ring. Large variations of the dynamics 
of the different carbon nuclei reside on these rings are also revealed by spin-lattice relaxation measurement.

\subsection{Spin-lattice Relaxation Measurements:}

The fluctuating local magnetic field experienced by the nucleus is the source of nuclear spin relaxation. The relaxation mechanism is governed by homonuclear dipole-dipole interaction, quadrupole interaction, chemical shift anisotropy interaction, and heteronuclear dipole-dipole interaction. For ${ }^{13} \mathrm{C}$ carbon major role in the relaxation mechanism is played by chemical shift anisotropy and heteronuclear dipole dipole interaction. The contribution of chemical shift anisotropy interaction is expressed as ${ }^{48-52}$

$$
\frac{1}{T_{1}^{C S A}}=\frac{2}{15} \gamma^{2} B^{2} S^{2}\left(\frac{\tau_{2}}{1+\omega^{2} \tau_{2}^{2}}\right)
$$

Where correlation time $\tau_{c}=3 \tau_{2}$ and $B$ is the applied magnetic field. Where $S^{2}=$ $(\Delta \delta)^{2}\left(1+\eta^{2} / 3\right)$ and $\left[\Delta \delta=\delta_{33}-\frac{\left(\delta_{22}+\delta_{11}\right)}{2}\right],\left(\eta=\frac{\delta_{22}-\delta_{11}}{\delta_{33}-\delta_{i s o}}\right)$.

The role of heteronuclear dipole dipole coupling on spin-lattice relaxation mechanism is articulated as ${ }^{52}$

$$
\begin{gathered}
\frac{1}{T_{1}^{D D}}=\frac{1}{10}\left(\frac{\gamma_{C} \gamma_{X} \hbar}{r_{C X}^{3}}\right)^{2} \tau_{2}\left[\frac{3}{1+\omega_{C}^{2} \tau_{2}^{2}}+\frac{1}{1+\left(\omega_{X}-\omega_{C}\right)^{2} \tau_{2}^{2}}\right. \\
\left.+\frac{6}{1+\left(\omega_{X}+\omega_{C}\right)^{2} \tau_{2}^{2}}\right]
\end{gathered}
$$

By keeping only the first term,

$$
\frac{1}{T_{1}^{D D}}=\frac{1}{10}\left(\frac{\gamma_{C} \gamma_{X} \hbar}{r_{C X}^{3}}\right)^{2} \tau_{2}\left[\frac{3}{1+\omega_{C}{ }^{2} \tau_{2}^{2}}\right]
$$

Where $\mathrm{X}$ represent hydrogen, oxygen and nitrogen atoms. $r_{C X}$ is the distance between carbon and neighbouring atoms hydrogen, oxygen, nitrogen. As the contribution of hetero-nuclear 
dipole-dipole interaction on spin-lattice relaxation mechanism is inversely proportional to the sixth power of the distance between the carbon and other nuclei, hence only the nearest neighbour distances is taken into consideration. Larmor precession frequency $\omega=2 \pi f=$ $2 \times 3.14 \times 125.758 \mathrm{MHz}=789.76024 \mathrm{MHz} ; B=11.74 T, \gamma_{C}=10.7084 \mathrm{MHz} / T, \gamma_{H}=$ $42.577 \mathrm{MHz} / T, \hbar=1.054 \times 10^{-34} \mathrm{Js}$.

The expression of the spin-lattice relaxation rate for ${ }^{13} \mathrm{C}$ carbon is

$$
\frac{1}{T_{1}}=\frac{1}{T_{1}^{C S A}}+\frac{1}{T_{1}^{D D}}=\frac{2}{15} \gamma^{2} B^{2} S^{2}\left(\frac{\tau_{2}}{1+\omega^{2} \tau_{2}^{2}}\right)+\frac{1}{10}\left(\frac{\gamma_{C} \gamma_{X} \hbar}{r_{C X}^{3}}\right)^{2} \tau_{2}\left[\frac{3}{1+\omega_{C}^{2} \tau_{2}^{2}}\right]
$$

Molecular correlation time is calculated by this equation.

Table 2: Spin-lattice relaxation time and molecular correlation time of cefpodoxime proxetil at crystallographically different carbon nuclei sites.

\begin{tabular}{|l|c|c|}
\hline \multicolumn{2}{|c|}{ Spin-lattice relaxation time and molecular correlation time at crystallographically } \\
different carbon nuclear sites of cefpodoxime proxetil \\
crystallographically & $\begin{array}{l}\text { Spin-lattice relaxation } \\
\text { time (s) }\end{array}$ & Molecular correlation \\
different carbon sites & time (s) \\
\hline C18 & $81 \pm 5$ & $8.2 \times 10^{-5}$ \\
\hline C8, C15 & $94 \pm 5$ & $1.6 \times 10^{-4}$ \\
\hline C6 & $112 \pm 5$ & $1.8 \times 10^{-4}$ \\
\hline C4 & $55 \pm 5$ & $6.9 \times 10^{-5}$ \\
\hline C1 & $82 \pm 5$ & $2.3 \times 10^{-4}$ \\
\hline C2 & $225 \pm 10$ & $2.2 \times 10^{-4}$ \\
\hline C11, C12 & $125 \pm 7$ & $2.6 \times 10^{-4}$ \\
\hline
\end{tabular}




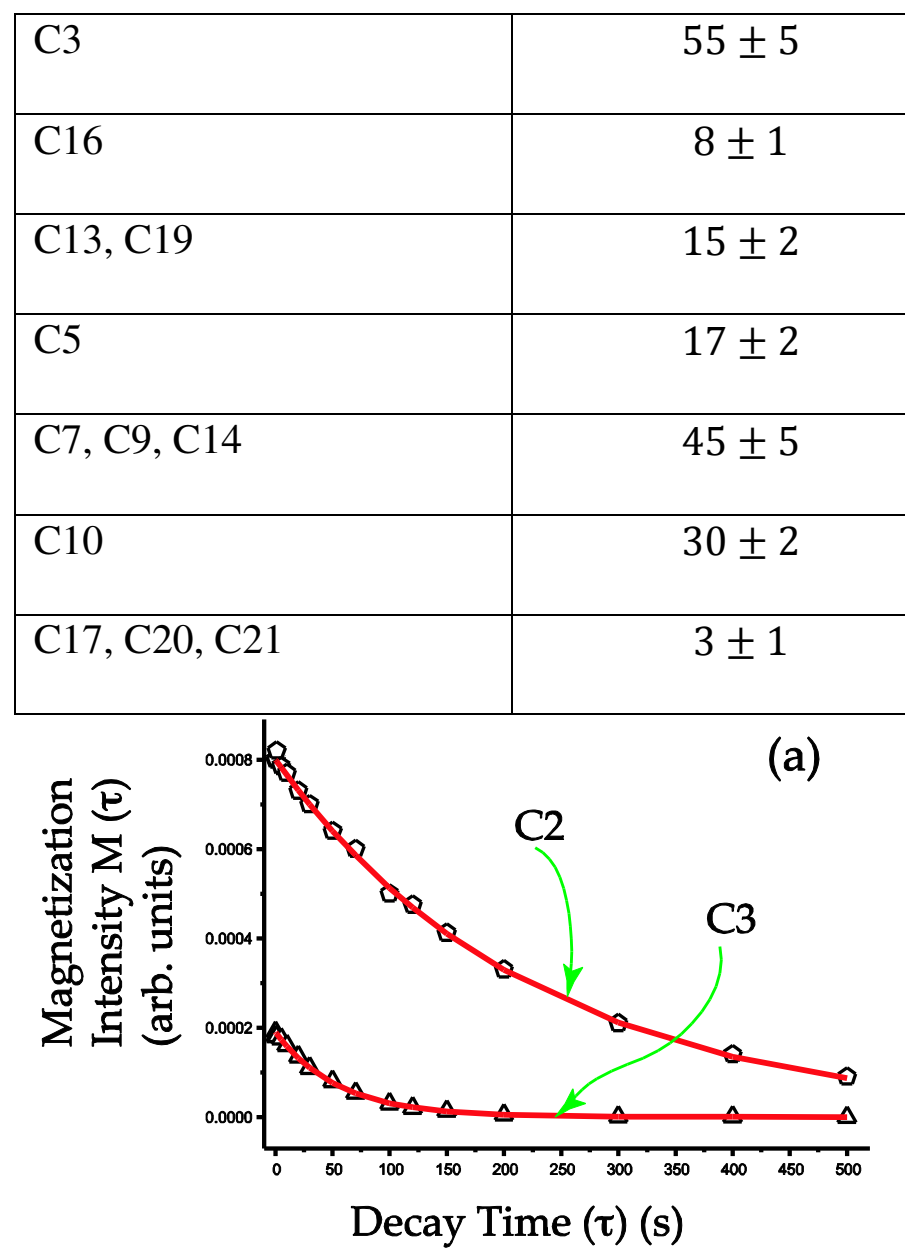

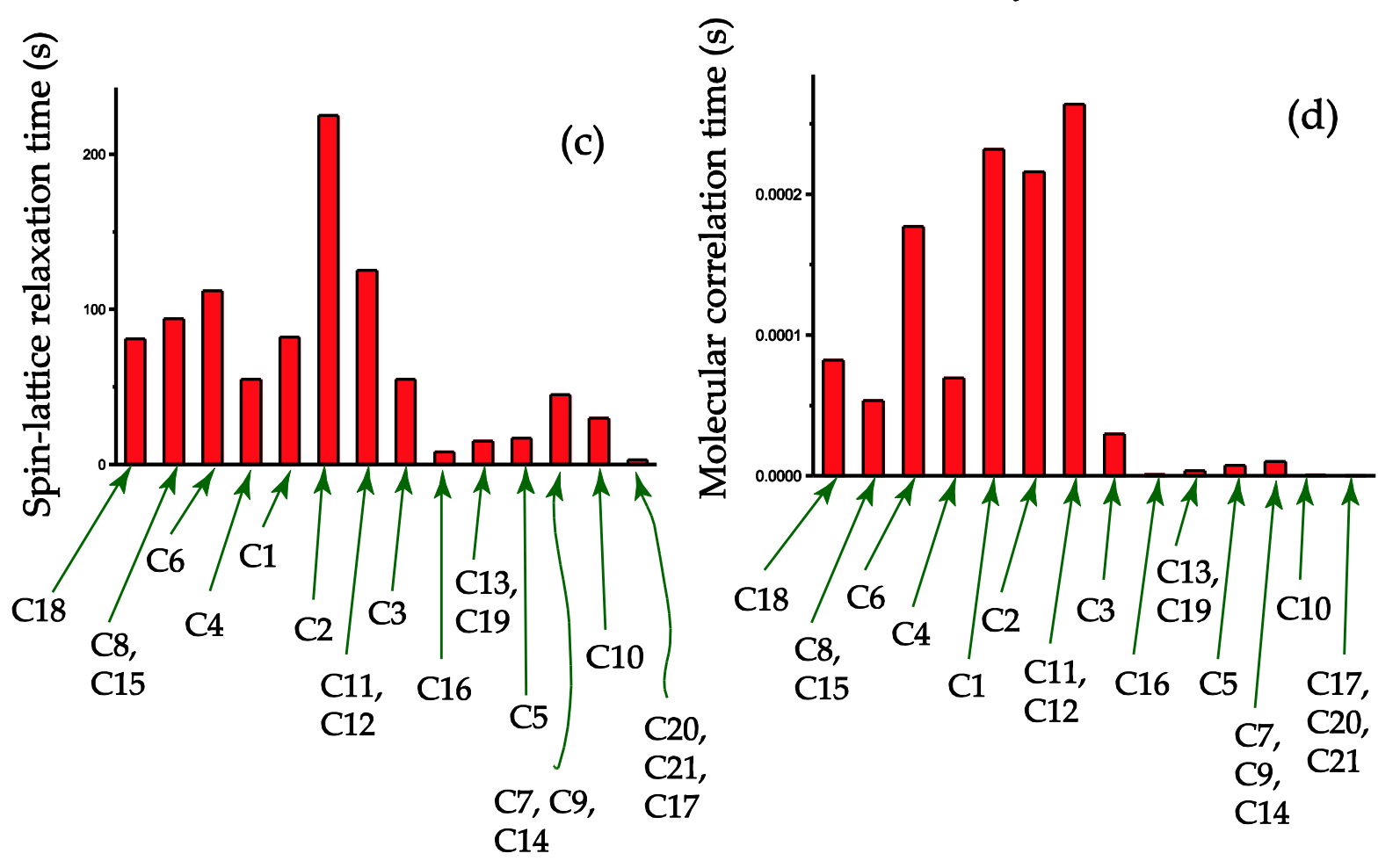


Figure 6: (a), (b) Longitudinal magnetization decay curve of cefpodoxime proxetil for $\mathrm{C}$, C3, C11, C12, C16. (c) Bar-diagram of spin-lattice relaxation time at chemically different carbon nuclei sites of cefpodoxime proxetil. (d) Bar-diagram of molecular correlation time at chemically different carbon nuclei sites of cefpodoxime proxetil.

Cefpodoxime proxetil is a prodrug of cefpodoxime and it falls in the cephalosporin family. The substitution at $\mathrm{C} 7$ position of the $\beta$-lactam ring (like acyl side chain, oxime group, and aminothiazole ring) is responsible for semi-synthetic incorporation. It also influences the antibacterial activity of the drug and it affects the binding affinity of the drug with $\beta$ lactamase. ${ }^{2}$ a huge variation of the spin-lattice relaxation time and molecular correlation time is observed in this region. The spin-lattice relaxation time of methyl group carbon C5 is $17 \mathrm{~s}$, whereas it is $225 \mathrm{~s}$ for $\mathrm{C} 2$ carbon resides on the aminothiazole ring. The molecular correlation time also varies in the range of $10^{-6} \mathrm{~s}$ to $10^{-4} \mathrm{~s}$. A remarkable variation of motional degrees of freedom is also observed among three carbon atoms $\mathrm{C} 1, \mathrm{C} 2$, and $\mathrm{C} 3$ lie on the aminothiazole ring, which improves the antibacterial activity of the drug. The spin lattice relaxation time of $\mathrm{C} 1, \mathrm{C} 2$, and $\mathrm{C} 3$ nuclei are respectively $82 \mathrm{~s}, 225 \mathrm{~s}$ and $55 \mathrm{~s}$. The molecular correlation time of $\mathrm{C} 1, \mathrm{C} 2$, and $\mathrm{C} 3$ nuclei are respectively $2.3 \times 10^{-4} \mathrm{~s}, 2.2 \times 10^{-4} \mathrm{~s}$, and $2.9 \times 10^{-5}$ s. Table 1 shows that the CSA parameters are also different for these three nuclei. ${ }^{55}$ The spin-lattice relaxation time of oxime group $\mathrm{C} 4$ nuclei is $55 \mathrm{~s}$ and it is $112 \mathrm{~s}$ for carbonyl group carbon C6. The CSA parameters of these two nuclei are not so different. Both C4 and C6 nuclei are in sp2 hybridized state, and double bonded with electronegative atoms nitrogen and oxygen respectively. The oxime and methyl group improve the stability of $\beta$ lactamases. ${ }^{55}$ The carbonyl group carbon $\mathrm{C} 8$ reside on the $\beta$-lactam ring is responsible for nucleophilic attack. The spin-lattice relaxation time and the molecular correlation time of C8 are respectively $94 \mathrm{~s}$ and $1.6 \times 10^{-4} \mathrm{~s}$. Figure 2 and Table 1 show that the spinning CSA sideband pattern of C8 is nearly axially symmetric and the CSA parameters are substantially 
large. The carboxylic acid group attached with $\mathrm{C} 12$ atom of dihydrothiazine ring is essential for PBPs affinity and it is also useful during the development of prodrug. It improves the permeability to the outer membrane of the bacterial cell. The spin-lattice relaxation time and the molecular correlation time of $\mathrm{C} 15$ nuclei reside on the carboxylic acid group are $94 \mathrm{~s}$ and $1.6 \times 10^{-4}$ S respectively. The CSA parameters are also substantially large for C15 nuclei. Ester group influence the pharmacokinetic behaviour of the prodrugs. It helps the polar groups to pass through the cell membranes and increase the bioavailability. ${ }^{2}$ The spin-lattice relaxation time and the molecular correlation time of the ester group carbon C18 are $81 \mathrm{~s}$ and $8.2 \times 10^{-5}$ s respectively. The isotropic chemical shift of C18 is largest among all the carbon nuclei of cefpodoxime proxetil molecule, and the CSA parameters are also very large. The spin-lattice relaxation time of the methyl group carbons $\mathrm{C} 20, \mathrm{C} 21$, and $\mathrm{C} 17$ is 3 s, lowest among all the nuclei of the molecule. It is possible to portray the structure activity relationship of a various molecular moiety of cefpodoxime proxetil by these types of investigations. These will definitely illuminate the path of developing the advanced antiviral drugs.

\section{Conclusion:}

Cefpodoxime proxetil is a prodrug of cefpodoxime, which has a broad spectrum of antibacterial activity encompassing both Gram-negative and Gram-positive bacteria. The structure and dynamics of cefpodoxime proxetil at atomic scale resolution is described by measuring CSA tensor, site-specific spin-lattice relaxation time, and calculating the molecular correlation time. The structure-activity relationship of various molecular moieties of the drug is also described by these measurements. Cefpodoxime proxetil molecule is associated with three rings, the aminothiazole ring, $\beta$-lactam ring, and the dihydrothiazine ring, which provide stability of the drug towards $\beta$-lactamases and increase the affinity of the 
drug to PBPs receptors. The anisotropy parameter $\left(\Delta \delta=\delta_{33}-\frac{\left(\delta_{11}+\delta_{22}\right)}{2}\right)$ of $\mathrm{C} 1$ carbon nuclei of the aminothiazole ring is much higher than $\mathrm{C} 2$ and $\mathrm{C} 3$ nuclei reside on the same ring. The spinning CSA sideband pattern of $\mathrm{C} 1$ is nearly axially symmetric, whereas the CSA pattern of $\mathrm{C} 2$ and $\mathrm{C} 3$ are highly asymmetric. A remarkable variation of motional degrees of freedom is also observed among three carbon nuclei $\mathrm{C} 1, \mathrm{C} 2$, and $\mathrm{C} 3$. Spin lattice relaxation time of C1, C2, and C3 nuclei are respectively $82 \mathrm{~s}, 225 \mathrm{~s}$, and 55s. The molecular correlation time of C1, C2, and C3 nuclei are respectively $2.3 \times 10^{-4} \mathrm{~s}, 2.2 \times 10^{-4} \mathrm{~s}$, and $2.9 \times 10^{-5} \mathrm{~s}$. The CSA parameters of $\mathrm{C} 8$ nuclei of $\beta$-lactam ring are much larger than $\mathrm{C} 7$ and $\mathrm{C} 9$ nuclei on the same ring. The spinning CSA sideband pattern of C8 is nearly axially symmetric, but the spinning CSA pattern of C7 and C9 are highly asymmetric. The carbonyl group carbon C8 is responsible for nucleophilic attack of the drug. The spin-lattice relaxation time and the molecular correlation time of $\mathrm{C} 8$ are respectively $94 \mathrm{~s}$ and $1.6 \times 10^{-4} \mathrm{~s}$, whereas the spinlattice relaxation time and the molecular correlation time of C7 and C9 are $45 \mathrm{~s}$ and $1.0 \times$ $10^{-5}$ s respectively. $\mathrm{C} 8$ are in sp2 hybridized state and $\mathrm{C} 7, \mathrm{C} 9$ are in sp3 hybridized state. The CSA parameters of $\mathrm{C} 11, \mathrm{C} 12$ nuclei of dihydrothiazine ring are much higher than $\mathrm{C} 10$ of the same ring. $\mathrm{C} 11, \mathrm{C} 12$ are in sp2 hybridized state, and $\mathrm{C} 10$ is in sp3 hybridized state. The spinning CSA sideband pattern of $\mathrm{C} 11$ and $\mathrm{C} 12$ are nearly axially symmetric and $\mathrm{C} 10$ is highly asymmetric. The spin-lattice relaxation time and the molecular correlation time of C11 and $\mathrm{C} 12$ are $125 \mathrm{~s}$ and $2.6 \times 10^{-4} \mathrm{~s}$ respectively, where as the spin-lattice relaxation time and the molecular correlation time of $\mathrm{C} 10$ are $30 \mathrm{~s}$ and $5.7 \times 10^{-7} \mathrm{~s}$ respectively. A large variation of CSA parameters and motional degrees of freedom are observed among carbon nuclei reside on these three rings, which implies that the electronic environment, molecular conformation, and molecular dynamics are altered substantially within the ring. The carboxylic acid group attached with $\mathrm{C} 12$ atom of dihydrothiazine ring is essential for PBPs affinity and it is also useful during the development of prodrug. It improves the permeability 
to the outer membrane of the bacterial cell. The spin-lattice relaxation time and the molecular correlation time of C15 nuclei reside on the carboxylic acid group are $94 \mathrm{~s}$ and $1.6 \times 10^{-4} \mathrm{~s}$ respectively. The CSA parameters are also substantially large for C15 nuclei. Ester group influence the pharmacokinetic behaviour of the prodrugs. It helps the polar groups to pass through the cell membranes and increase the bioavailability. The spin-lattice relaxation time and the molecular correlation time of the ester group carbon $\mathrm{C} 18$ are $81 \mathrm{~s}$ and $8.2 \times 10^{-5} \mathrm{~s}$ respectively. The isotropic chemical shift of C18 is largest among all the carbon nuclei of cefpodoxime proxetil molecule, and the CSA parameters are also very large. The spin-lattice relaxation time of the methyl group carbons C20, C21 and C17 is 3s, the lowest among all the nuclei of the molecule. The substitution at $\mathrm{C} 7$ position of the $\beta$-lactam ring (like acyl side chain, oxime group and aminothiazole ring) is responsible for semi-synthetic incorporation. It also influences the antibacterial activity of the drug and it affects the binding affinity of the drug with $\beta$-lactamase. A huge variation of the spin-lattice relaxation time and molecular correlation time is observed in this region. The spin-lattice relaxation time of methyl group carbon $\mathrm{C} 5$ is $17 \mathrm{~s}$, whereas it is $225 \mathrm{~s}$ for $\mathrm{C} 2$ carbon resides on the aminothiazole ring. The

molecular correlation time also varies in the range of $10^{-6} \mathrm{~s}$ to $10^{-4} \mathrm{~s}$. These types of description of the structure-activity relationship of the antibacterial drug cefpodoxime proxetil will definitely enlighten the path of developing the advanced antibacterial drugs. It will also enrich the data of NMR crystallography of the drug molecules.

\section{Acknowledgements}

The author Manasi Ghosh is indebted to Science and Engineering Research Board (SERB), Department of Science and Technology (DST), government of India (File no. EMR/2016/000249) for financial support.

\section{Reference:}


1. G. R. Donowitz and G. L. Mandell, Beta-Lactam Antibiotics, Medical Intelligence Drug therapy, The New England Journal of Medicine, 1988, 318, 419-426.

2. A. E. Gamal, A. E. Mostafa, H. Yazeed, H. Al-Otaibi, Abdullah A. Al-Badr, Chapter One-Cefpodoxime proxetil, Profiles of Drug Substances, Excipients and Related Methodology, 2019, 44, 1-165.

3. B. J. Wylie, C. M. Rienstra, Multidimensional solid state NMR of anisotropic interactions in peptides and proteins, The Journal of Chemical Physics, 2008, 128, 052077-1 - 052077-16.

4. A. Shoji, S. Ando, S. Kuroki, I. Ando, G.A. Webb, Annu. Rep. NMR Spectrosc., 1993, 26, 55-98.

5. W.S. Veeman, Carbon-13 Chemical Shift Anisotropy, Progress in Nuclear Magnetic Resonance Spectroscopy, 1984, 16, 193-235.

6. L. Shao, J.J. Titman, Chemical Shift Anisotropy Amplification, Progress in Nuclear Magnetic Resonance Spectroscopy, 2007, 51, 103-137.

7. G. Wu, Solid-state $17 \mathrm{O}$ NMR studies of organic and biological molecules, Progress in Nuclear Magnetic Resonance Spectroscopy, 2008, 52, 118-169.

8. I. Ando, T. Asakura (Eds.), Solid State NMR of Polymers, Elsevier Science, Amsterdam, 1998.

9. O.N. Antzutkin, in: M.J. Duer (Ed.), Solid-state NMR Spectroscopy: Principles and Applications, Blackwell Sciences, Oxford, 2002, p. 280.

10. A. Ramamoorthy (Ed.), NMR Spectroscopy of Biological Solids, CRC Press, Cleveland, 2005.

11. B.C. Gerstein, C.R. Dybowski, Transient Techniques in NMR of Solids, An Introduction to Theory and Practice, Academic Press, Orlando, 1985.

12. O.N. Antzutkin, Sideband manipulation in magic-angle-spinning nuclear magnetic resonance, Progress in Nuclear Magnetic Resonance Spectroscopy, 1999, 35, 203-266.

13. T.M. Duncan, A Compilation of Chemical Shift Anisotropics, The Farragut Press, Chicago, 1990

14. A.C. de Dios, Ab initio calculations of the NMR chemical shift, Progress in Nuclear Magnetic Resonance Spectroscopy, 1996, 29, 229-278

15. S. Sen, Dynamics in Inorganic Glass-forming Liquids by NMR Spectroscopy, Progress in Nuclear Magnetic Resonance Spectroscopy, 2019,116, 155-176.

16. D. C. Kaseman, T. Endo, and S. Sen. Structural disorder and the effects of aging in a phosphate glass: Results from two-dimensional ${ }^{31} \mathrm{P}$ PASS NMR spectroscopy, Journal of Non-Crystalline Solids 2013, 359, 33-39.

17. D. C. Kaseman, O. Gulbiten, B. G. Aitken, and S. Sen, Isotropic rotation vs. shear relaxation in supercooled liquids with globular cage molecules, The Journal of Chemical Physics 2016, 144, 174501.

18. D. D. Laws, Hans-Marcus L. Bitter, and A. Jerschow, Solid-State NMR Spectroscopic Methods in Chemistry, Angewandte Chemie Internationak Edition 2002, 41, 3096 3129.

19. M. J. Duer, Solid state NMR spectroscopy principles and applications, Blackwell science, 2007. 
20. M. M. Miyachiro, C. Contreras-Martel, and A. Dessen, Penicillin-binding proteins (PBPS) and bacterial cell wall elongation complexes, Sub-Cellular Biochemistry, 2019, 93, 273-289. doi:10.1007/978-3-030-28151-9_8. ISBN 978-3-030-281502. PMID $\underline{31939154}$

21. B. Kasten, and R. Reski, $\beta$-Lactam antibiotics inhibit chloroplast division in a moss (Physcomitrella patens) but not in tomato (Lycopersicon esculentum), Journal of Plant Physiology, 1997, 150 (1), 137-140. doi:10.1016/S0176-1617(97)80193-9

22. J. F. Fisher, S. O. Meroueh, and S. Mobashery, Bacterial resistance to $\beta$-lactam antibiotics: compelling opportunism, compelling opportunity, Chemical Reviews, 2005, 105 (2), 395-424. doi:10.1021/cr030102i. PMID 15700950.

23. U. Haeberlen, High Resolution NMR in Solids: Selective Averaging. Academic Press : New York, 1976.

24. R. Tycko, G. Dabbagh, and P. A. Mirau, Determination of chemical shift anisotropy lineshapes in a two-dimensional magic angle spinning NMR experiment, Journal of Magnetic Resonance 1989, 85, 265-274.

25. S. F. Liu, J. D. Mao, and K. Schmidt-Rohr, A robust technique for two-dimensional separation of undistorted chemical shift anisotropy powder patterns in magic angle spinning NMR, Journal of Magnetic Resonance 2002, 155,15-28.

26. J. C. C. Chan, and R. Tycko, Recoupling of chemical shift anisotropies in solid state NMR under high speed magic angle spinning and in uniformly ${ }^{13} \mathrm{C}$ labelled systems, Journal of Chemical Physics 2003, 118,8378-8389.

27. G. Hou, L. Byeon In-Ja, J. Ahn, A. M. Gronenborn, and T. Polenova, Recoupling of chemical shift anisotropy by R-symmetry sequences in magic angle spinning NMR spectroscopy, Journal of Chemical Physics 2012, 137,134201-134210.

28. AD Bax, N. M. Szeverenyi, and G. E. Maciel, Chemical shift anisotropy in powdered solids studied by 2D FT NMR with flipping of the spinning axis, Journal of Magnetic Resonance 1983, 55, 494-497.

29. AD Bax, N. M. Szeverenyi, and G. E. Maciel, Correlation of isotropic shifts and chemical shift anisotropies by two-dimensional Fourier-transform magic angle hopping NMR spectroscopy, Journal of Magnetic Resonance 1983, 52, 147-152.

30. AD Bax, N. M. Szeverenyi, and G. E. Maciel, Chemical shift anisotropy in powdered solids studied by 2D FT CP/MAS NMR, Journal of Magnetic Resonance 1983, 51, 400408.

31. Z. Gan, High-resolution chemical shift and chemical shift anisotropy correlation in solids using slow magic angle spinning, Journal of American Chemical Society 1992, 114, 8307-8309.

32. W. T. Dixon, Spinning-sideband-free and spinning-sideband-only NMR spectra in spinning samples, The Journal of Chemical Physics, 1982, 77, 1800-1809.

33. O. N. Antzutkin, S. C. Shekar and M. H. Levitt, Two-dimensional sideband separation in magic angle spinning NMR, Journal of Magnetic Resonance A, 1995, 115, 7-19.

34. M. Ghosh, S. Sadhukhan and K. K. Dey, Elucidating the internal structure and dynamics of $\alpha$-chitin by 2DPASS-MAS-NMR and spin-lattice relaxation measurements, Solid State Nuclear Magnetic Resonance, 2019, 97, 7-16.

35. M. Ghosh, B. P. Prajapati, N. Kango and K. K. Dey, A comprehensive and comparative study of the internal structure and dynamics of natural $\beta$-keratin and regenerated $\beta$ keratin by solid state NMR spectroscopy, Solid State Nuclear Magnetic Resonance, 2019, 101, 1-11. 
36. M. Ghosh, N. Kango and K. K. Dey, Investigation of the internal structure and dynamics of cellulose by ${ }^{13} \mathrm{C}-\mathrm{NMR}$ relaxometry and 2DPASS-MAS-NMR measurements, Journal of Biomolecular NMR, 2019, 73, 601-616.

37. K. K. Dey and M. Ghosh, Understanding the effect of deacetylation on chitin by measuring chemical shift anisotropy tensor and spin lattice relaxation time, Chemical Physics Letters, 2020, 738, 136782.

38. K. K. Dey, S. Gayen and M. Ghosh, Investigation of the detailed internal structure and dynamics of itraconazole by solid-state NMR measurements, ACS Omega, 2019, 4, 21627-21635.

39. K. K. Dey, S. Gayen and M. Ghosh, Understanding the correlation between structure and dynamics of clocortolone pivalate by solid state NMR measurement, RSC Advances, 2020, 10, 4310-4321.

40. K. K. Dey and M. Ghosh, Understanding the Structure and Dynamics of Antiinflammatory Corticosteroid Dexamethasone by solid state NMR Spectroscopy, RSC Advances, 2020, 10, 37564.

41. K. K. Dey and M. Ghosh, Determination of the Correlation between the Structure and Dynamics of Deflazacort by solid state NMR measurements, New Journal of Chemistry, 2020 , 44, 18419-18430.https://doi.org/10.1039/D0NJ03418E.

42. K. K. Dey and M. Ghosh, Determination of Chemical Shift Anisotropy Tensor and Molecular Correlation Time of Proton Pump Inhibitor Omeprazole by Solid State NMR Measurements, New Journal of Chemistry, 2020, 44, 19393-19403 https://doi.org/10.1039/D0NJ01827A.

43. B. J. Walder, K. K. Dey, D. C. Kaseman, J. H. Baltisberger and P. J. Grandinetti, Sideband separation experiments in NMR with phase incremented echo train acquisition, The Journal of Chemical Physics, 2013, 138,174203-1-174203-12.

44. S. Sen, Dynamics in Inorganic Glass-forming Liquids by NMR Spectroscopy, Progress in Nuclear Magnetic Resonance Spectroscopy (2019),116, 155-176.

45. D. C. Kaseman, T. Endo, S. Sen. Structural disorder and the effects of aging in a phosphate glass: Results from two-dimensional 31P PASS NMR spectroscopy, Journal of Non-Crystalline Solids 2013, 359, 33-39.

46. D. C. Kaseman, O. Gulbiten, B. G. Aitken, S. Sen, Isotropic rotation vs. shear relaxation in supercooled liquids with globular cage molecules, The Journal of Chemical Physics 2016, 144, 174501.

47. D. A. Torchia, The measurement of proton-enhanced carbon-13 T1 values by method which suppresses artifacts, The Journal of Magnetic Resonance, 1978, 30, 613.

48. M. P. Nicholas, E. Eryilmaz, F. Ferrage, D. Cowburn and R. Ghose, Nuclear spin relaxation in isotropic and anisotropic media, Progress in Nuclear Magnetic Resonance Spectroscopy, 2010, 57, 111-158.

49. A. M. Orendt and J. C. Facelli, Solid state effects on NMR chemical shifts, Annual Report NMR Spectroscopy, 2007, 62, 115-178.

50. N. Tjandra, A. Szabo and Ad. Bax, Protein backbone dynamics and 15B chemical shift anisotropy from quantitative measurement of relaxation interference effects, Journal of American Chemical Society, 1996, 118(29), 6986 - 6991.

51. P. Dais and A. Spyros, ${ }^{13} \mathrm{C}$ nuclear magnetic relaxation and local dynamics of synthetic polymers in dilute solution and in the bulk state, Progress in Nuclear Magnetic Resonance Spectroscopy, 1995, 27, 555-633. 
52. F. A. L. Anet and D. J. O'Leary, The shielding tensor Part II: Understanding its strange effect on relaxation, Concepts in Magnetic Resonance, 1992, 4, 35-52.

53. H. M. McConnell, Theory of Nuclear Magnetic Shielding in Molecules: Long-Range Dipolar Shielding of protons, The Journal of Chemical Physics, 1957, 27, 226

54. R. J. Abraham, M. Mobli, and Smith R.J., 1H chemical shifts in NMR : Carbonyl anisotropies and steric effect in aromatic aldehydes and ketones, Magnetic Resonance in Chemistry, 2003, 41, 26-36.

55. G. G. Zhanel, A. R. Golden, S. Zelenitsky, K. Wiebe, C. K. Lawrence, H. J. Adam, T. Idowu, R. Domalaon, F. Schweizer, M. A. Zhanel, P. R. S. Lagacé-Wiens, A. J. Walkty, A. Noreddin, J. P. Lynch III, J. A. Karlowsky, Cefderocol: A Siderophore Cephalosporin with Activity Against Carbapenem-Resistant and Multidrug-Resistant Gram-Negative Bacilli, Drugs 2019, 79, 271-289.

56. J. Herzfeld and A. E. Berger, Sideband intensities in NMR spectra of samples spinning at the magic angle, Journal of Chemical Physics, 1980, 73, 6021-6030.

57. K. K. Dey, M. Ghosh, Investigation of the Structure and Dynamics of Antiviral Drug Adefovir Dipivoxil by Site-Specific Spin-Lattice Relaxation Time Measurements and Chemical Shift Anisotropy Tensor Measurements, ACS Omega, 2020, 5, 29373https://doi.org/10.1021/acsomega.0c04205

58. R. Bhowal, A. Balaraman, M. Ghosh, S. Dutta, K. K. Dey, D. Chopra, Probing Atomistic Behavior to Unravel Dielectric Phenomena in Charge Transfer Cocrystals, Journal of American Chemical Society 2020, https://dx.doi.org/10.1021/jacs.0c11459 

Figures
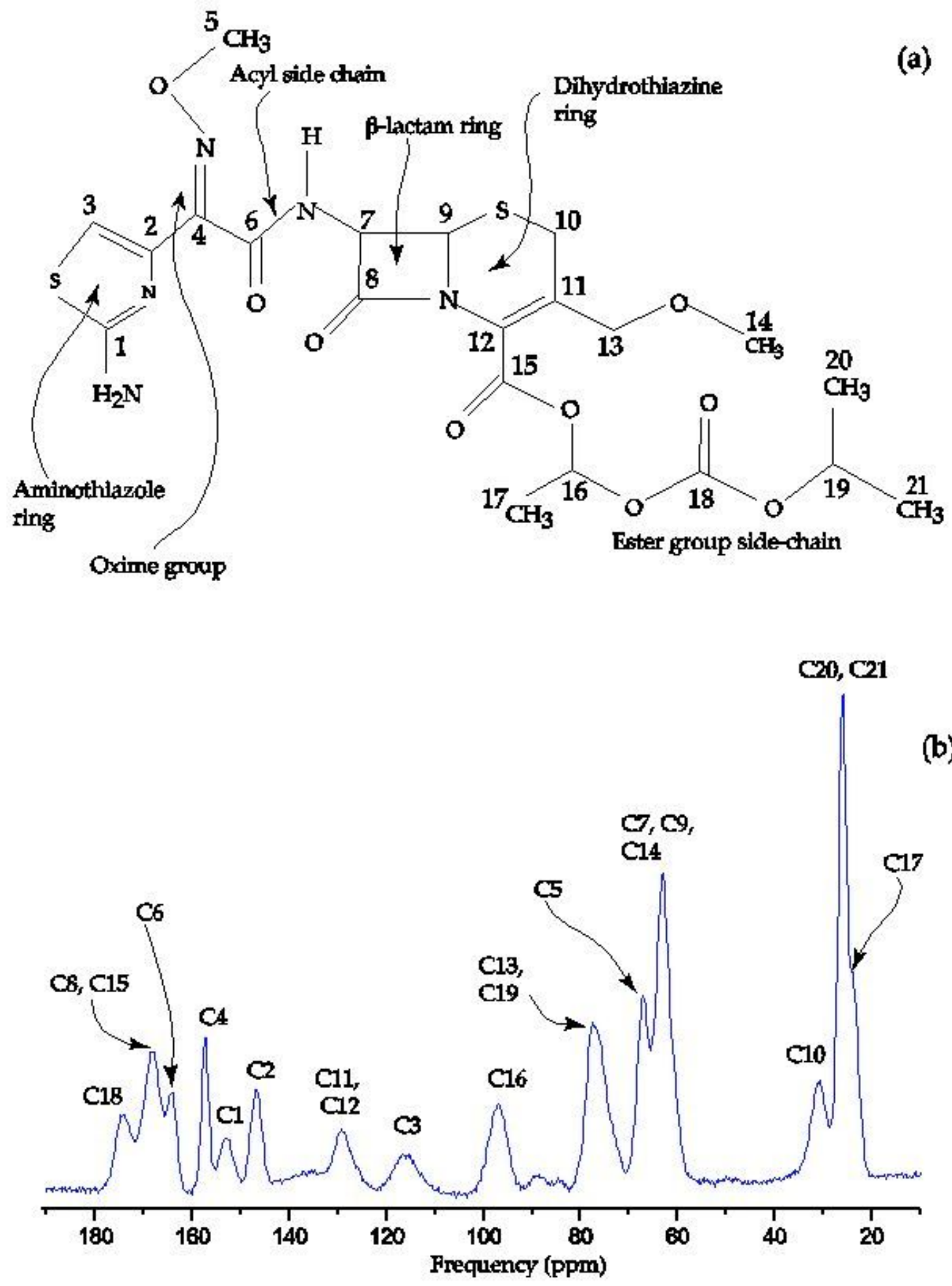

Figure 1

(a) The chemical structure of cefpodoxime proxetil, (b) 13C CP-MAS SSNMR spectrum of cefpodoxime proxetil. 


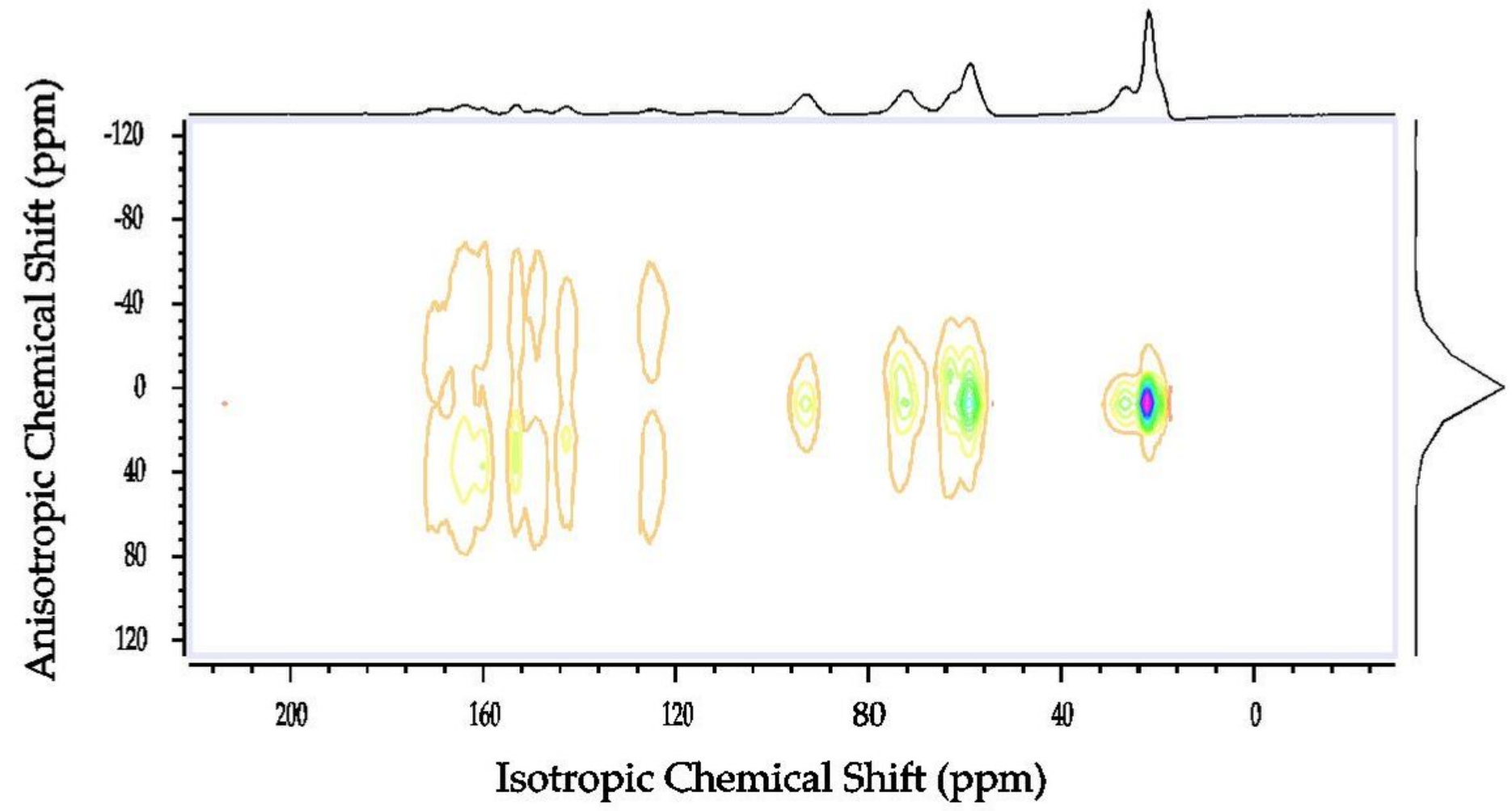

Figure 2

2DPASS CP-MAS NMR spectrum of cefpodoxime proxetil. The direction dimension represents the pure isotropic spectrum of cefpodoxime proxetil with zero sideband. 

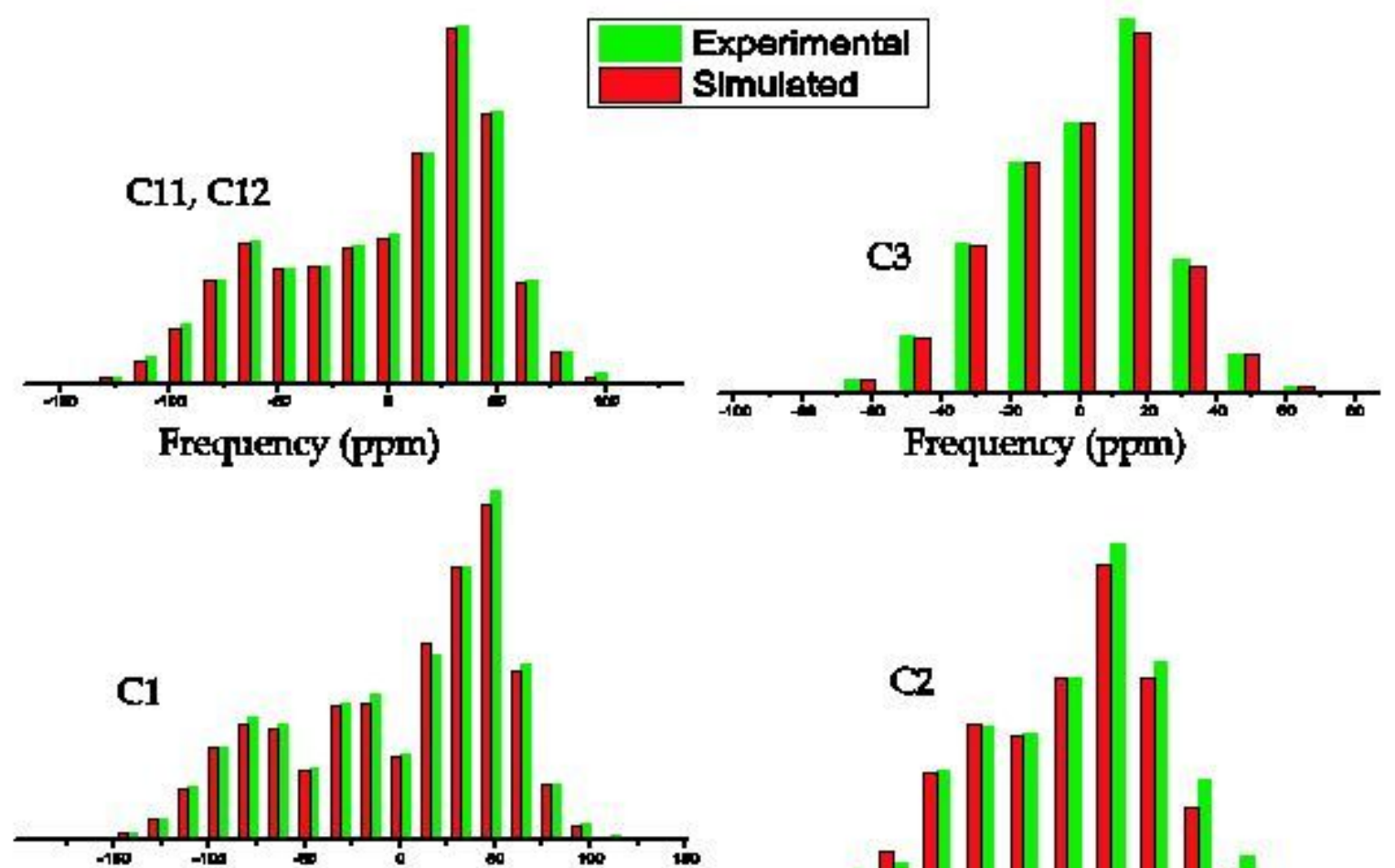

Frequency (ppm)

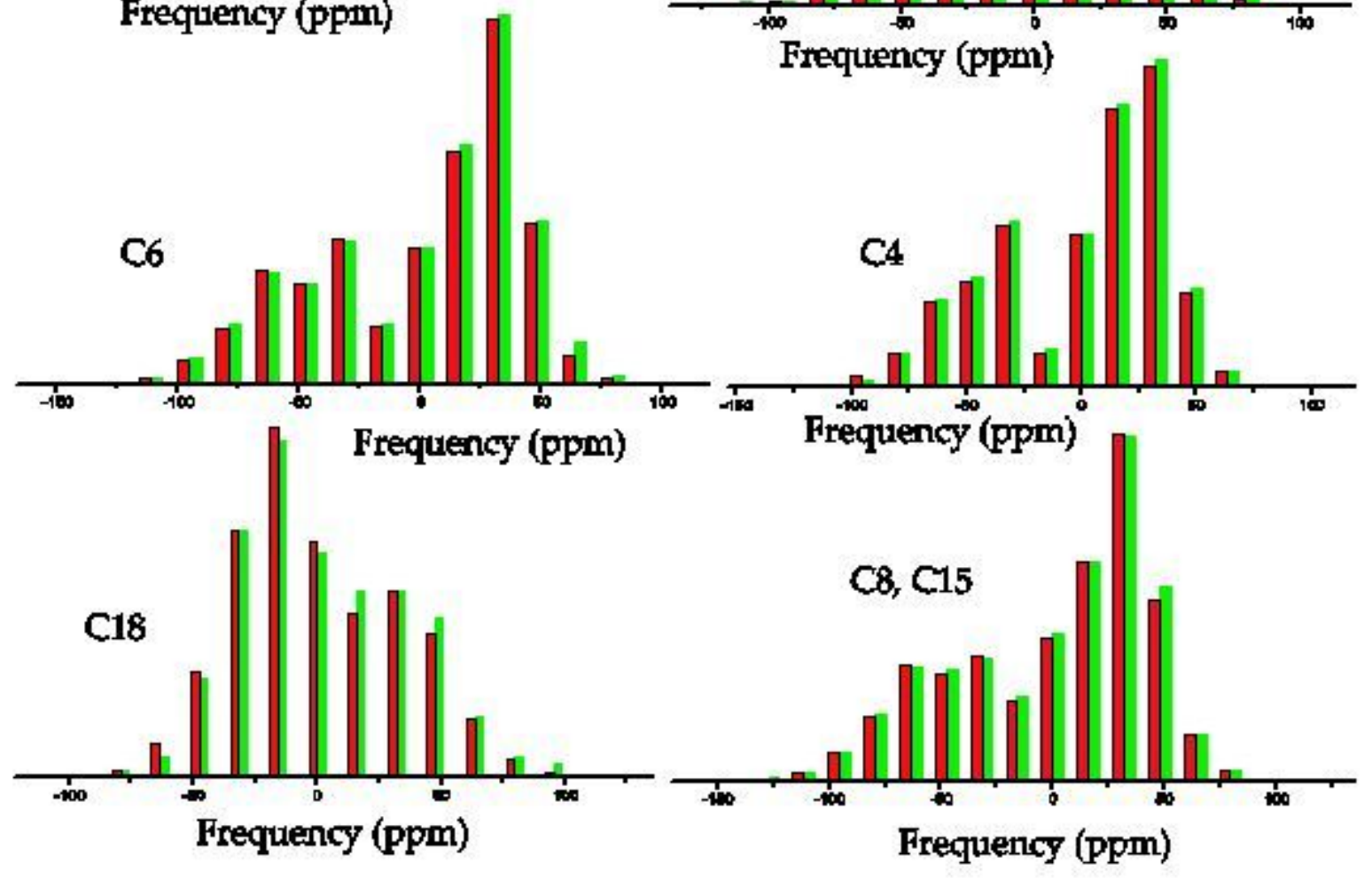

Figure 3

Spinning CSA sideband pattern of cefpodoxime proxetil. 


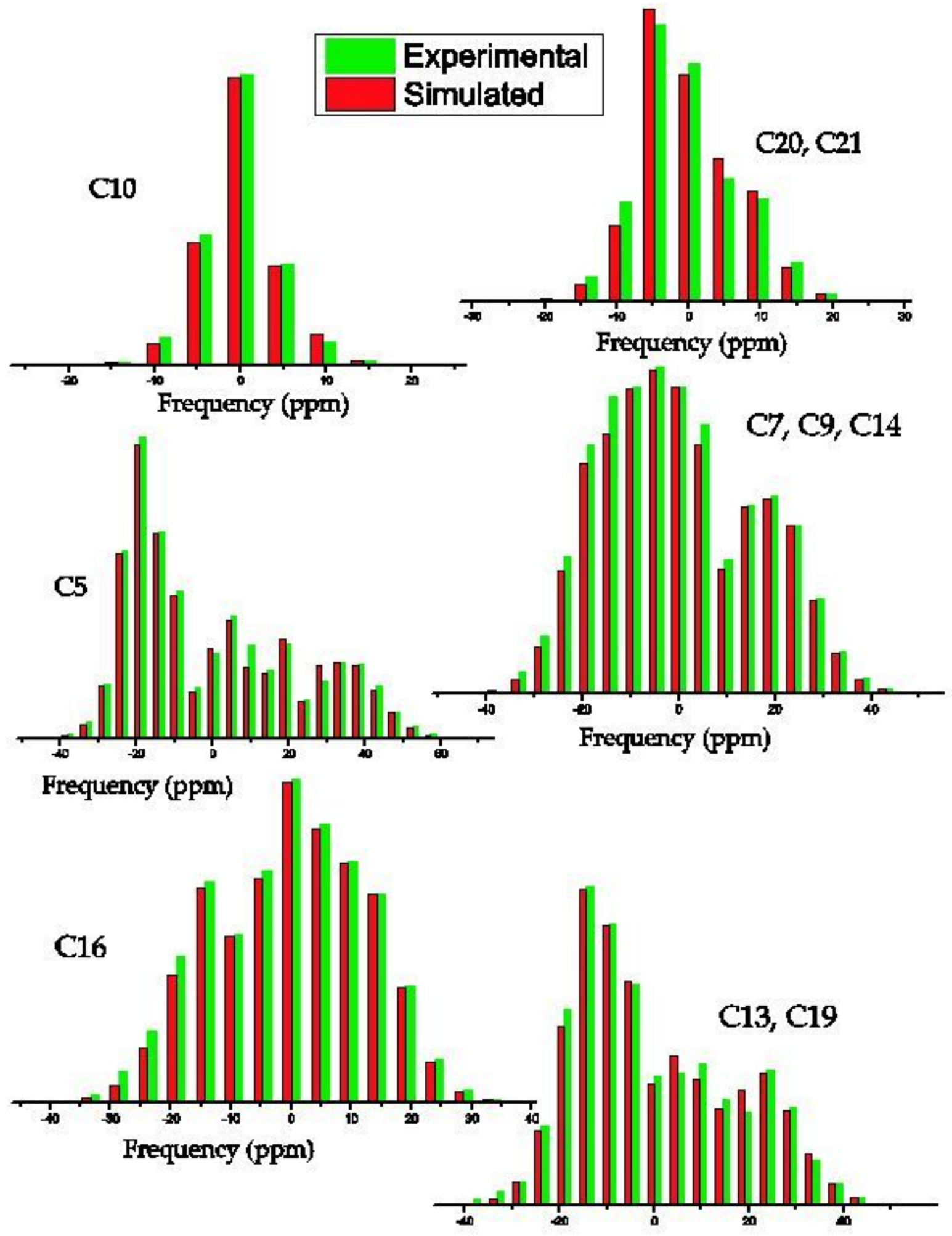

Figure 4

Spinning CSA sideband pattern of cefpodoxime proxetil. 

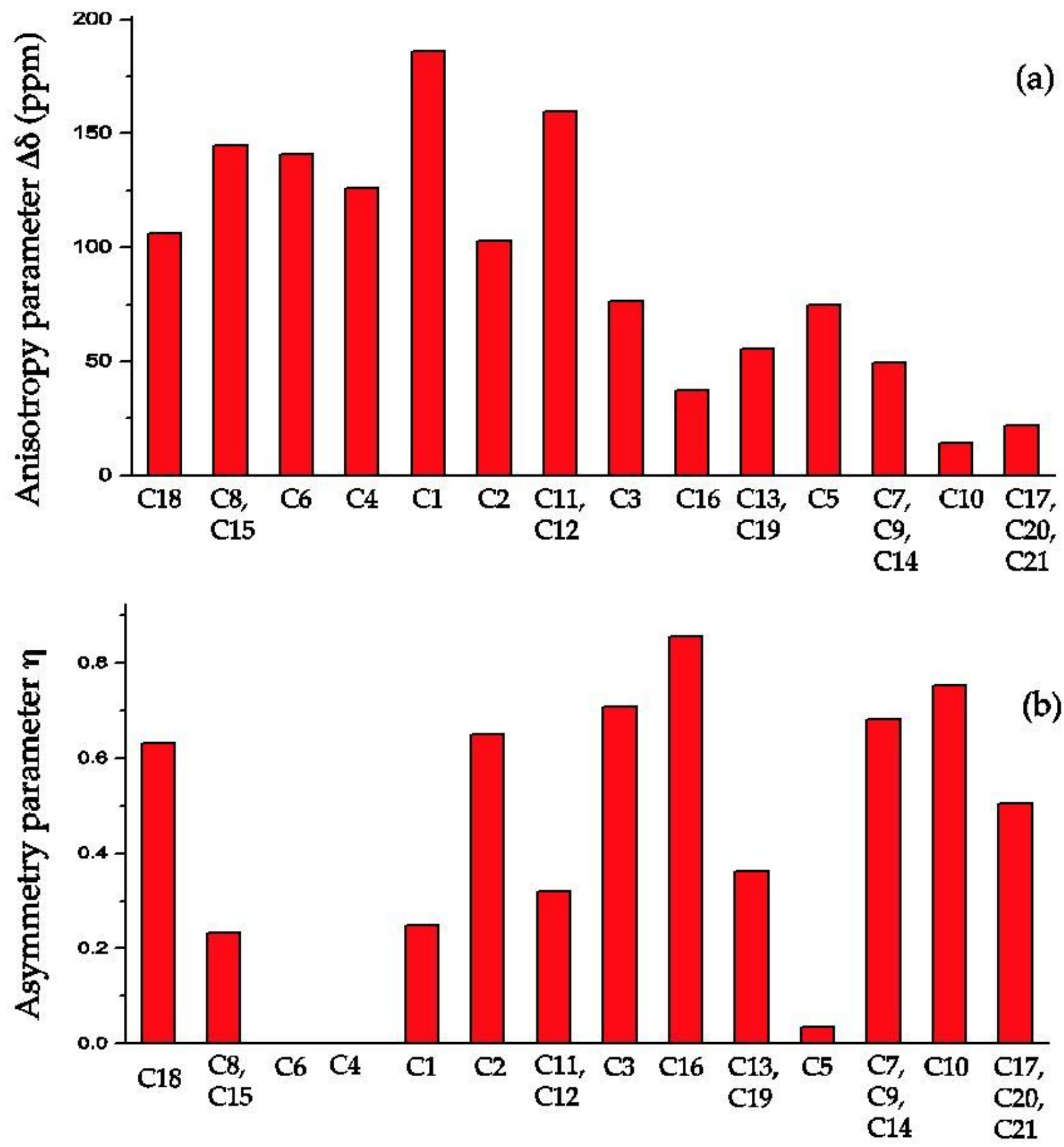

Figure 5

The bar-diagram of (a) anisotropy parameter and (b) asymmetry parameter of cefpodoxime proxetil at crystallographically different carbon sites. 

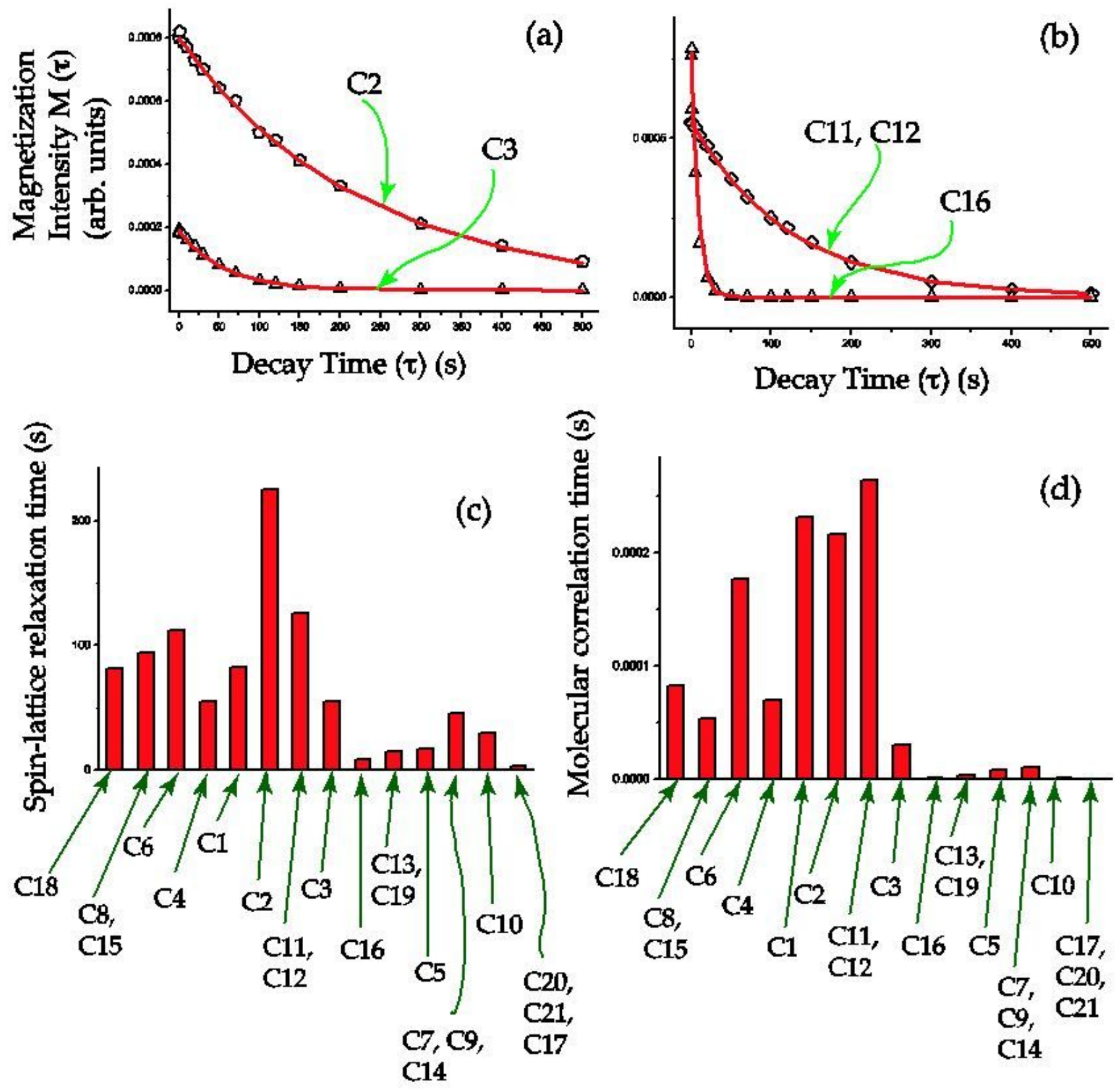

Figure 6

(a), (b) Longitudinal magnetization decay curve of cefpodoxime proxetil for $\mathrm{C} 2, \mathrm{C} 3, \mathrm{C} 11, \mathrm{C} 12, \mathrm{C} 16$. (c) Bardiagram of spin-lattice relaxation time at chemically different carbon nuclei sites of cefpodoxime proxetil. (d) Bar-diagram of molecular correlation time at chemically different carbon nuclei sites of cefpodoxime proxetil. 